\title{
Foam Transport in Porous Media - A Review
}

\author{
ZF Zhang \\ VL Freedman \\ L Zhong
}

November 2009

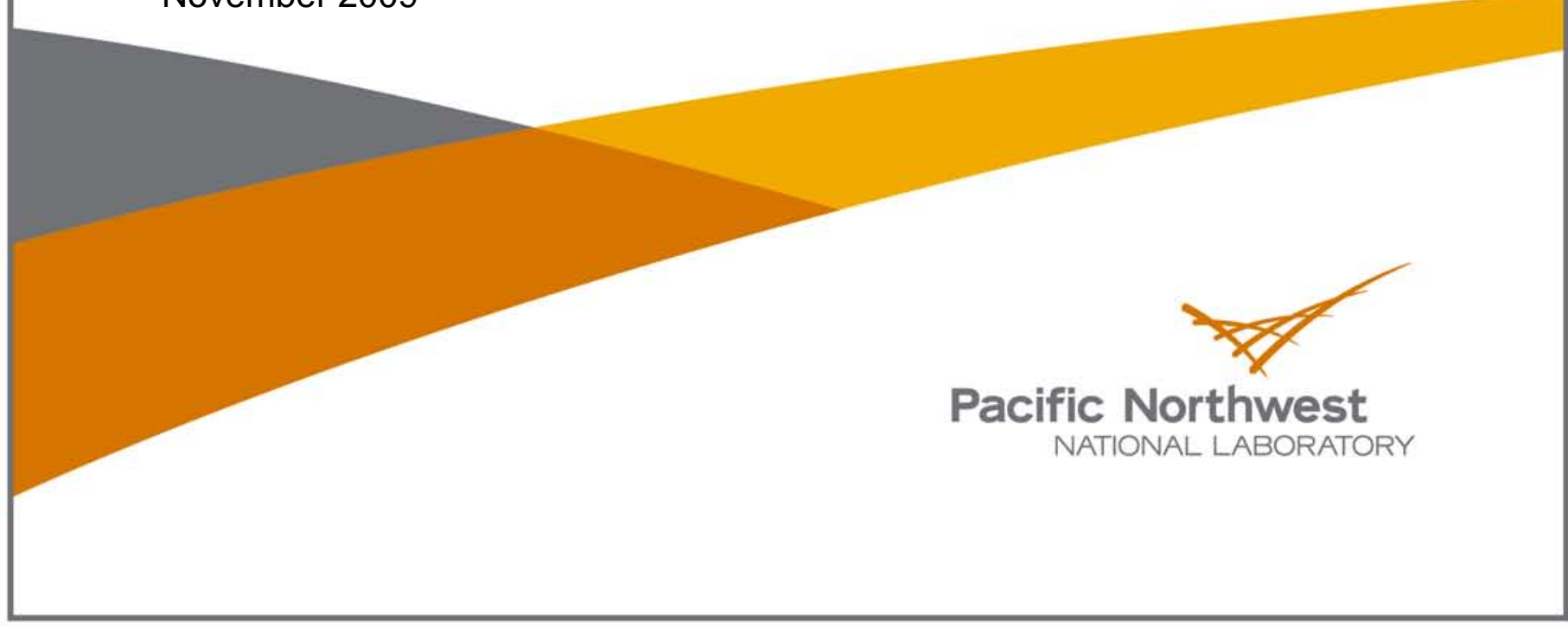




\title{
DISCLAIMER
}

This report was prepared as an account of work sponsored by an agency of the United States Government. Neither the United States Government nor any agency thereof, nor Battelle Memorial Institute, nor any of their employees, makes any warranty, express or implied, or assumes any legal liability or responsibility for the accuracy, completeness, or usefulness of any information, apparatus, product, or process disclosed, or represents that its use would not infringe privately owned rights. Reference herein to any specific commercial product, process, or service by trade name, trademark, manufacturer, or otherwise does not necessarily constitute or imply its endorsement, recommendation, or favoring by the United States Government or any agency thereof, or Battelle Memorial Institute. The views and opinions of authors expressed herein do not necessarily state or reflect those of the United States Government or any agency thereof.

\author{
PACIFIC NORTHWEST NATIONAL LABORATORY \\ operated by \\ BATTELLE \\ for the \\ UNITED STATES DEPARTMENT OF ENERGY \\ under Contract DE-AC05-76RL01830 \\ Printed in the United States of America \\ Available to DOE and DOE contractors from the \\ Office of Scientific and Technical Information, \\ P.O. Box 62, Oak Ridge, TN 37831-0062; \\ ph: (865) 576-8401 \\ fax: (865) 576-5728 \\ email: reports@adonis.osti.gov \\ Available to the public from the National Technical Information Service, \\ U.S. Department of Commerce, 5285 Port Royal Rd., Springfield, VA 22161 \\ ph: (800) 553-6847 \\ fax: (703) 605-6900 \\ email: orders@ntis.fedworld.gov \\ online ordering: http://www.ntis.gov/ordering.htm
}




\title{
Foam Transport in Porous Media - A Review
}

\author{
ZF Zhang \\ VL Freedman \\ L Zhong
}

November 2009

Prepared for

the U.S. Department of Energy

under Contract DE-AC05-76RL01830

Pacific Northwest National Laboratory

Richland, Washington 99352 



\section{Executive Summary}

Amendment solutions with or without surfactants have been used to remove contaminants from soil. However, they have drawbacks in that amendment solutions often mobilize the plume, and its movement is controlled by gravity and preferential flow paths. Foam is an emulsion-like, two-phase system in which gas cells are dispersed in a liquid and separated by thin liquid films called lamellae. The potential advantages of using foams in sub-surface remediation include providing better control on the volume of fluids injected, uniformity of contact, and the ability to contain the migration of contaminant-laden liquids. It is expected that foam can serve as a carrier of amendments for vadose zone remediation, e.g., at the Hanford Site. As part of the U.S. Department of Energy's EM-20 program, a numerical simulation capability will be added to the Subsurface Transport Over Multiple Phases (STOMP) flow simulator. The primary purpose of this document is to review the modeling approaches of foam transport in porous media. However, as an aid to understanding the simulation approaches, some experiments under unsaturated conditions and the processes of foam transport are also reviewed.

Foam may be formed when the surfactant concentration is above the critical micelle concentration. There are two main types of foams -ball foam (microfoam) and polyhedral foam. The characteristics of bulk foam are described by properties such as foam quality, texture, stability, density, surface tension, disjoining pressure, etc. Foam has been used to flush contaminants such as metals, organics, and nonaqueous phase liquids from unsaturated soil. Ball foam, or colloidal gas aphrons, reportedly have been used for soil flushing in contaminated site remediation and was found to be more efficient than surfactant solutions on the basis of weight of contaminant removed per gram of surfactant. Experiments also indicate that the polyhedral foam can be used to enhance soil remediation.

The transport of foam in porous media is complicated in that the number of lamellae present governs flow characteristics such as viscosity, relative permeability, fluid distribution, and interactions between fluids. Hence, foam is a non-Newtonian fluid. During transport, foam destruction and formation occur. The net result of the two processes determines the foam texture (i.e., bubble density). Some of the foam may be trapped during transport. According to the impacts of the aqueous and gas flow rates, foam flow generally has two regimes - weak and strong foam. There is also a minimum pressure gradient to initiate foam flow and a critical capillary for foam to be sustained. Similar to other fluids, the transport of foam is described by Darcy's law with the exception that the foam viscosity is variable.

Three major approaches to modeling foam transport in porous media are the empirical, semi-empirical, and mechanistic methods. Mechanistic approaches can be complete in principle but may be difficult for obtaining reliable parameters, whereas empirical and semi-empirical approaches can be limited by the detail used to describe foam rheology and mobility. Mechanistic approaches include the bubble population-balance model, the network/percolation theory, the catastrophe theory, and the filtration theory. All these methods were developed for modeling polyhedral foam, with the exception that the filtration theory method was developed for modeling ball foam (microfoam). 



\section{Acknowledgments}

Funding for this work was provided by the U.S. Department of Energy's EM-22 project managed by Dawn M. Wellman and EM-20 project managed by Mark D. Freshley. 



\section{Acronyms and Abbreviations}

$\begin{array}{ll}\text { ATF } & \text { automatic transmission fluid } \\ \text { CBF } & \text { common black films } \\ \text { CGA } & \text { colloidal gas aphrons } \\ \text { CMC } & \text { critical micelle concentration } \\ \text { DNAPL } & \text { dense non-aqueous phase liquid } \\ \text { MRF } & \text { mobility reduction factor } \\ \text { NBF } & \text { Newton black films } \\ \text { NAPL } & \text { non-aqueous phase liquid } \\ \text { PCP } & \text { Pentachlorophenol } \\ \text { TCE } & \text { Trichloroethylene }\end{array}$



Roman Letters:

\section{Symbols}

\begin{tabular}{|c|c|}
\hline $\begin{array}{l}a_{L} \\
a, b, c\end{array}$ & $\begin{array}{l}\text { Langmuir constant } \\
\text { constants }\end{array}$ \\
\hline$A_{s}$ & intermediate variable \\
\hline$B$ & backbone fraction in the percolation model \\
\hline$C$ & surfactant concentration \\
\hline$C_{m}$ & mean curvature of a lamella \\
\hline $\mathrm{C}_{\mathrm{s}}, \mathrm{C}_{\mathrm{s}}^{0}$ & aqueous concentration of surfactant \\
\hline$C_{g}, C_{c}$ & constants for foam generation and coalesce \\
\hline$c_{f}$ & constant \\
\hline $\mathrm{d}_{\mathrm{b}}, \mathrm{d}_{\mathrm{p}}$ & particle diameter \\
\hline$d_{g}$ & diameter of the spherical grain of filter media (collector) \\
\hline $\mathrm{d}_{\mathrm{t}}$ & tube diameter \\
\hline$D$ & a constant or dispersion coefficient \\
\hline$D_{w}$ & diffusivity of water \\
\hline$e_{s}, e_{o}, e_{v}$ & exponents \\
\hline$E$ & a constant \\
\hline$f$ & foam quality, (-) \\
\hline$f^{*}$ & a specific foam quality that divides the high and low foam quality \\
\hline$f_{b}$ & fraction of bonds in the percolation theory \\
\hline$f_{b}^{0}$ & fraction of bonds without surfactant \\
\hline$f_{b}^{c}$ & percolation threshold \\
\hline $\mathrm{f}_{\mathrm{c}}, \mathrm{f}_{\mathrm{k}}$, and $\mathrm{f}_{\mathrm{p}}$ & functions \\
\hline$f_{s n}$ & fraction of throat blocked \\
\hline$f_{w}$ & fractional flow of water \\
\hline$f_{w}$ & slope of the $f_{w}$ vs. $S_{w}$ curve \\
\hline$F$ & percolation fraction \\
\hline$g$ & gravity constant \\
\hline$G$ & conductivity in the percolation model \\
\hline$G_{p}$ & pressure gradient \\
\hline$G_{p}^{\min }$ & minimum pressure gradient to keep foam moving \\
\hline$h$ & film thickness \\
\hline$H$ & the Hamaker constant, usually assumed to be $10^{-20} \mathrm{~J}$ \\
\hline$k$ & permeability \\
\hline$k_{a}$ & pseudo first-order rate coefficient for attachment \\
\hline$k_{b}$ & the Boltzmann constant \\
\hline$k_{r}$ & relative permeability \\
\hline$k_{r f}$ & relative permeability of foam \\
\hline$k_{r g}$ & relative permeability of gas without foam \\
\hline$k_{r w}$ & relative permeability of the aqueous phase \\
\hline$k_{r n w}$ & relative permeability of the nonaqueous phase \\
\hline $\mathrm{k}_{1}$ & generation rate constant \\
\hline $\mathrm{k}_{-1}$ & coalescence rate constant \\
\hline
\end{tabular}




\begin{tabular}{|c|c|}
\hline$k_{-1}{ }^{0}$ & scaling constant \\
\hline K & effective hydraulic conductivity \\
\hline$l$ & the length of a single pore \\
\hline$L_{s}$ & length of liquid slugs between the bubbles \\
\hline $\mathrm{L}_{\mathrm{BD}}$ & dimensionless bubble length \\
\hline$L_{b}$ & average cluster length \\
\hline $\mathrm{m}_{l}$ & mass of liquid in foam \\
\hline$n_{f}$ & bubble density or foam texture \\
\hline$n_{f}^{\max }$ & maximum bubble density or foam texture \\
\hline$n_{f f}$ & flowing foam bubble density \\
\hline$n_{f t}$ & trapped foam bubble density \\
\hline $\bar{n}$ & average number of pores in a gas channel blocked by a single pore throat \\
\hline$n_{L}$ & number of lamellae per unit length \\
\hline$N_{R}, N_{p e}, N_{G}$ & dimensionless numbers \\
\hline$N_{L o}$ & \\
\hline $\mathrm{N}_{\mathrm{s}}$ & dimensionless bubble group \\
\hline$N_{c}$ & capillary number \\
\hline$P$ & pressure \\
\hline$P_{c}$ & capillary pressure \\
\hline$P_{c}^{c r}$ & the critical capillary pressure \\
\hline$P_{c}^{\text {rup }}$ & rupture capillary pressure corresponding to $\mathrm{P}_{\mathrm{d}}^{\text {rup }}$ \\
\hline$P_{d}$ & disjoining pressure \\
\hline$P_{d}^{e l}$ & disjoining pressure due to electrostatic repulsion \\
\hline $\mathrm{P}_{\mathrm{d}}^{\mathrm{vw}}$ & disjoining pressure van der Waals attraction \\
\hline$P_{d}^{\text {sh }}$ & disjoining pressure steric/hydration forces \\
\hline$P_{d}^{\max }$ & maximum disjoining pressure \\
\hline$P_{d}^{\text {rup }}$ & the disjoining pressure at which the film ruptures \\
\hline$q$ & Darcy velocity/flux \\
\hline$q_{g}$ & gas Darcy velocity/flux \\
\hline$q_{w}$ & aqueous Darcy velocity/flux \\
\hline$q_{f}$ & foam Darcy velocity/flux \\
\hline$q_{w}$ & nonaqueous Darcy velocity/flux \\
\hline$q_{t}$ & total flux of the aqueous and nonaqueous phases \\
\hline Q & source/since term \\
\hline$r$ & net change of $n_{\mathrm{f}}$ per unit time \\
\hline$r_{g}, r_{c}$ & bubble generation and coalescence rates \\
\hline$r_{c}$ & radius of curvature of plateau border in foam lamella \\
\hline$r_{\text {cap }}$ & capillary radius \\
\hline$r_{b}$ & foam bubble radius \\
\hline$P_{f}$ & foam pressure \\
\hline$R$ & ideal gas constant or retardation factor \\
\hline$R_{f}$ & expansion factor of foam \\
\hline$S$ & saturation \\
\hline$S_{i}$ & $\begin{array}{l}\text { saturation corresponding to the point a tangent line is drawn in the } f_{w} \text { vs. } S_{w} \\
\text { curve }\end{array}$ \\
\hline$S_{f}$ & foam saturation \\
\hline
\end{tabular}




$\begin{array}{ll}S_{f f} & \text { flowing foam saturation } \\ S_{f t} & \text { trapped foam saturation } \\ S_{f t}{ }^{\text {max }} & \text { maximum trapped foam saturation } \\ S_{g} & \text { gas saturation } \\ S_{g r} & \text { residual gas saturation } \\ S_{i} & \text { initial saturation } \\ S_{n w} & \text { nonaqueous phase saturation } \\ S_{o} & \text { oil saturation } \\ S_{w} & \text { aqueous saturation } \\ S_{w}{ }^{*} & \text { limiting aqueous saturation } \\ S_{w c} & \text { connate water saturation } \\ S_{w r} & \text { residual aqueous saturation } \\ t & \text { time } \\ T & \text { absolute temperature } \\ U & \text { approach velocity } \\ U_{p} & \text { buoyant rise velocity of the bubbles } \\ V_{g} & \text { gas velocity } \\ V_{w} & \text { aqueous velocity } \\ V_{f} & \text { foam velocity } \\ V_{g} & \text { volume of gas in foam } \\ V_{l} & \text { volume of liquid in foam } \\ \mathrm{V}_{\mathrm{f}} & \text { bulk volume of foam } \\ x & \text { spatial variable }\end{array}$

Greek Letters:

$\begin{array}{ll}\alpha & \text { model parameter } \\ \alpha_{\mathrm{L}} & \text { longitudinal dispersivity } \\ \gamma_{\mathrm{B}} & \text { the exponent for the scaling law } \\ \chi_{f} & \text { fraction of foam trapped } \\ \chi_{f}^{\text {max }} & \text { maximum fraction of trapped foam } \\ \lambda & \text { filter coefficient } \\ \lambda_{\mathrm{w}} & \text { water mobility } \\ \lambda_{\mathrm{w}}{ }^{*} & \text { water mobility at critical saturation } \\ \eta & \text { model parameter } \\ \beta & \text { a trapping parameter ( } \mathrm{L}^{-3} \text { ) } \\ \phi & \text { porosity } \\ \kappa_{f} & \text { specific conductivity of foam } \\ \kappa_{l} & \text { specific conductivity of liquid } \\ \mu_{f} & \text { foam effective viscosity } \\ \mu_{g} & \text { viscosity of gas without foam } \\ \mu_{1}, \mu_{2}, \mu_{3}, \mu_{4} & \text { components of viscosity } \\ \mu_{w} & \text { viscosity of the aqueous phase } \\ \rho_{f} & \text { density of a foam } \\ \rho_{l} & \text { density of liquid }\end{array}$




$\begin{array}{ll}\sigma & \text { surface tension or interfacial tension } \\ \sigma_{0} & \text { surface tension without surfactant } \\ \xi & \text { interfacial area increased coefficient } \\ \xi_{1} & \text { geometrical factor } \\ \Gamma_{\infty} & \text { maximum surface excess concentration } \\ \Gamma_{s} & \text { the amount of surfactant adsorption on porous media surfaces } \\ \nabla P_{0} & \text { a model parameter } \\ \Delta \mathrm{P}^{\max } & \text { maximum pressure drop }\end{array}$

Subscripts

$\begin{array}{ll}\gamma & \text { any phase } \\ \mathrm{w} & \text { aqueous phase } \\ \mathrm{nw} & \text { nonaqueous phase } \\ \mathrm{g} & \text { gas phase } \\ \mathrm{f} & \text { foam } \\ \mathrm{o} & \text { oil } \\ \mathrm{t} & \text { total or trapped } \\ \mathrm{ff} & \text { flowing foam } \\ \mathrm{ft} & \text { foam trapped }\end{array}$




\section{Contents}

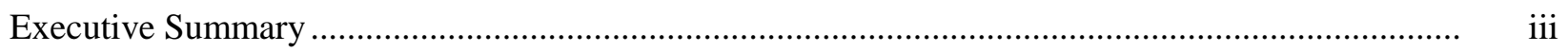

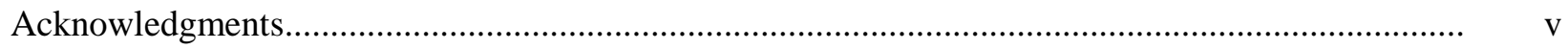

Acronyms and Abbreviations ..................................................................................................... vii

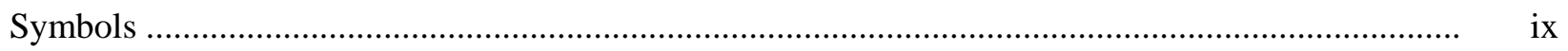

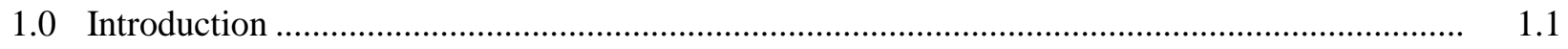

2.0 Properties of Bulk Foam............................................................................................ 2.1

2.1 General Properties .................................................................................................... 2.1

2.2 Surface Tension of Surfactant Solution........................................................................ 2.3

2.3 Foam Disjoining Pressure ............................................................................................. 2.5

3.0 Vadose Zone Remediation with Foam ......................................................................

3.1 Remediation with Micro-Foam …...................................................................................... 3.1

3.2 Remediation with Polyhedral Foam .......................................................................... 3.2

4.0 Foam Transport Processes in Porous Media......................................................................

4.1 Foam Dynamic Processes........................................................................................

4.1.1 Foam Destruction ........................................................................................ 4.1

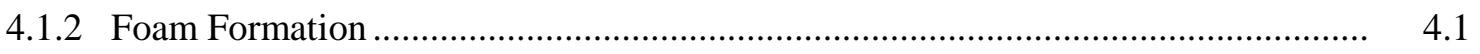

4.1.3 Foam Entrapment .......................................................................................... 4.1

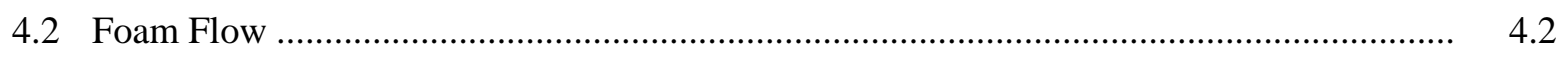

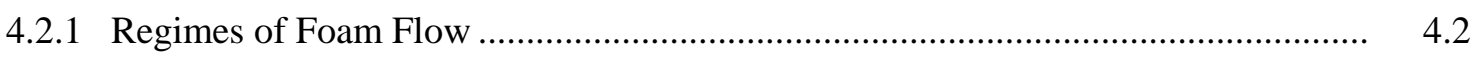

4.2.2 Minimum Pressure Gradient ................................................................................... 4.3

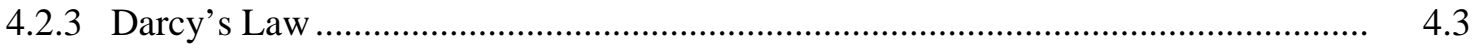

4.2.4 Limiting Capillary Pressure............................................................................ 4.3

4.2.5 Effective Viscosity of Foam................................................................................ 4.5

4.2.6 Effective Mobility of Foam ................................................................................ 4.6

5.0 Modeling Foam Transport ......................................................................................... 5.1

5.1 Empirical Methods ............................................................................................... 5.1

5.1.1 Mobility Reduction Factor Approach............................................................... 5.1

5.1 .2 Effective Viscosity Approach ...................................................................... 5.3

5.2 The Semi-Empirical Fractional Flow Theory ................................................................. 5.3

5.2.1 The Fractional Flow Model for a Oil-Water System............................................. 5.4

5.2.2 The Fractional Flow Model for a Foam-Water System.......................................... 5.5

5.2.3 The Fractional Flow Model for the Gas-Oil-Water System .................................... 5.7

5.2.4 Fraction Flow Model Limitations......................................................................... 5.9

5.3 Mechanistic Methods ................................................................................................. 5.10

5.3.1 Bubble Population-Balance Model ..................................................................... 5.10

5.3 .2 Catastrophe Theory …................................................................................. 5.14 


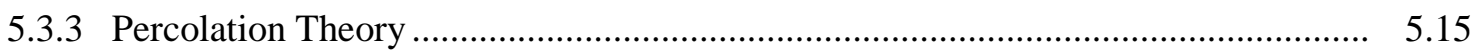

5.3.4 The Filtration Theory for Ball Foam.................................................................. 5.18

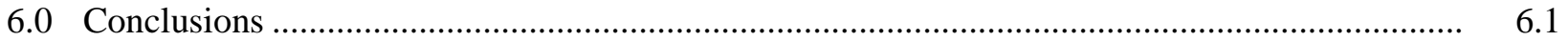

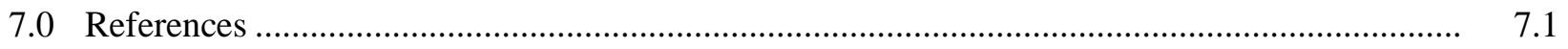

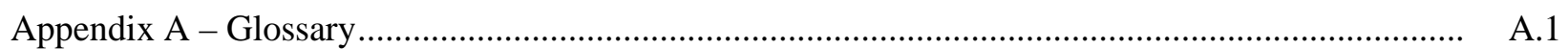




\section{Figures}

2.1 Diagram of the Surface Tension Variation with the Surfactant Concentration .......................... 2.4

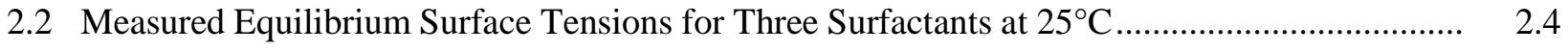

2.3 Idealized Disjoining Pressure Isotherm ............................................................................... 2.6

3.1 Schematic Illustration of the Use of Foams to Remediate Contaminated Soil ........................... 3.3

3.2 Schematic Drawing of Soil and Simulated Fracture Zones in a Cylindrical Bench-Scale Chamber Used for Foam Flow Tests .................................................................... 3.3

4.1 Concept of the Gas-Water, Two-Phase Flow and Weak and Strong Foams in Oorous Media

4.2 Feedback Mechanism Near the Limiting Capillary Pressure, $\mathrm{P}_{\mathrm{c}}$

\section{Tables}

2.1 Foamability Comparison of Different Surfactants.

2.2 Critical Micelle Concentration, Saturation Surface Excess Concentration, and Langmuir Constant for Surfactants at the Water/Air Interface at $25^{\circ} \mathrm{C}$

3.1 Summary of Surfactant Foam Enhanced Soil Remediations. 



\subsection{Introduction}

Subsurface delivery of amendments in the unsaturated zone has been used for in situ remediation applications such as soil-washing and surfactant-flooding processes. However, drawbacks to delivering amendments to heterogeneous porous media, with or without surfactants, include difficulty in containing amendments to the treatment zone and the potential for desorbed or surfactant-dissolved contaminants migrating to groundwater (Hanson et al. 1993; Dresel et al. 2008). Because preferential flow paths of the solution in the vadose zone may result, the amendment solution often bypasses the less permeable zones. The infiltration of amendment solution is predominantly controlled by gravity, leading to a dominant downward spreading of the contaminant plume. As a result, surfactant-flooding operations have not been particularly effective in removing the contaminants from soils (Peters et al. 1992). Moreover, surfactantflooding operations can be costly, and soil pores may plug due to the biodegradation of surfactants (Allred and Brown 1994; Luthy et al. 1994; Vignon and Rubin 1989).

Unlike the aqueous solution, gas movement in the vadose zone does not have downward dominance due to its low density. Hence, to overcome the problems caused by using a surfactant solution, new in situ remediation techniques using gas or a gas-like amendment carriers are being sought so that contaminants are immobilized while the carrier has the enhanced horizontal, but no preferential, vertical migration. However, gas flow also tends to bypass the less permeable zone.

Foam, however, is a potential medium that can be used to effectively distribute amendments to the deep vadose zone for contaminant remediation. Foam is defined as a two-phase system in which gas bubbles are dispersed in a liquid and separated by thin liquid films called lamellae (Bikerman 1973). A major attraction of foam is the possibility of spontaneously diverting flow from high-permeability to lowpermeability layers. Other potential advantages of using foams for subsurface remediation are that it can provide better control on the fluids injected and uniformity of contact. This occurs because, under at least some conditions, stronger foams form spontaneously and hence block the flow channels in highpermeability media. Whereas the gas component of foam is compressible, the liquid component can generally be considered incompressible. Thus, the pressure gradient within foam often is the primary driving force of foam flow, and there is no significant gravity-caused flow.

Foam has been shown to improve reservoir sweep efficiency in gas-injection enhanced oil recovery projects (Hirasaki 1989a, b; Smith 1988). In enhanced oil recovery operations, because foam has an effective viscosity much higher than that of gas, it can reduce viscous fingering and gravity override caused by injecting gas, supercritical $\mathrm{CO}_{2}$ or steam. An extensive body of literature exists on the use of foam to enhance oil recovery. Thorough reviews can be found in Marsden (1986), Liu and Brigham (1992), and in Kovscek and Radke (1994).

Foam has been used to flush contaminants such as metals, organics, and nonaqueous phase liquids (NAPLs) from unsaturated soil (Mulligan and Eftekhari 2003; Wang and Mulligan 2004; Rothmel et al. 1998; Peters et al. 1994). In-situ soil flushing involves the use of an appropriate surfactant solution to dissolve or emulsify contaminants and bring them to the surface for disposal/destruction. The selection of a foam formulation depends on several factors that are often site-specific. The factors include the potential loss of surfactant by adsorption on soil particles, the loss of foam formation and stability due to contaminant dissolution, and the pressure required to inject the foam. 
Development of an amendment foam delivery technology is currently being considered for in situ remediation of vadose zone metals and radionuclides at the Hanford Site. This technology development is a multiple-step process, one of which examines several physical aspects of foam delivery. Although laboratory and field experiments are an important part of investigating foam delivery in the unsaturated zone, the ability to simulate foam transport is pertinent to the development of the remediation technology. A simulation capability will be important for extrapolating to conditions outside experimental conditions, and for making field predictions of foam and contaminant transport.

Because foam delivery has been used in the petroleum industry to enhance oil recovery since the 1960s, several models of foam transport in porous media have been developed for application to enhance oil recovery. However, all of these approaches have been developed for initially saturated conditions. An amendment foam delivery technology at Hanford is targeted at recalcitrant contaminants residing in the deep vadose zone. Hence, model development will differ from existing approaches because the sediments are unsaturated initially.

The primary purpose of this document is to review the modeling approaches of foam transport in porous media. However, as an aid to understanding the simulation approaches, some experiments under unsaturated conditions and the processes of foam transport are also reviewed. Properties of bulk foam are introduced in Section 2, followed by a summary of remediation experiments using foam in Section 3, and a description of foam processes in Section 4. Section 5 provides a comprehensive review of the modeling approaches of foam transport in the subsurface. For convenience, relevant terms are summarized in the Appendix. 


\subsection{Properties of Bulk Foam}

Bulk foam is the foam for which the length scale of the confining space is greater than the length scale of the foam bubbles (Schramm 2005). Bulk foam is often divided into kugelschaum ("ball foam”) and polyderschaum ("polyhydral foam") (Bikerman 1973). The former consists of well-separated, spherical bubbles, while the latter consists of polyhedral bubbles separated by thin-liquid films called lamellae. In a porous medium, the bubbles and lamellae generally span completely across pores, and the foam is designated as confined foam. Although the foam in porous media is different from bulk foam, the properties of foam are often characterized by those of bulk foam. Hence, this section describes some properties of bulk foam.

\subsection{General Properties}

\section{Critical Micelle Concentration}

Critical micelle concentration (CMC) is the surfactant concentration above which molecular aggregates, termed micelles, begin to form. Some typical molar CMC values for low electrolyte concentration at room temperature are $10^{-5}-10^{-4} \mathrm{M}$ for non-ionics surfactants, $10^{-3}-10^{-2} \mathrm{M}$ for anionics surfactants, and $10^{-3}-10^{-1} \mathrm{M}$ for amphoterics surfactants (Schramm 2005, p. 83). Compilations of CMC values are given in Rosen (1989) and Mukerjee and Mysels (1971).

\section{Foam Quality}

Foam quality, $\mathrm{f}$, is the volume fraction of gas in foam:

$$
f=\frac{V_{g}}{V_{l}+V_{g}}
$$

where $\mathrm{Vg}$ is the volume of gas in foam, and $\mathrm{Vl}$ is the volume of liquid in foam. Foam quality is often expressed in percentage and sometimes referred as the "Mitchell foam quality."

\section{Foam Texture}

Foam texture, $n$, is defined as the number of lamellae per unit volume. Although foam texture is of prime importance in foam rheology, there are currently no reliable experimental techniques to measure it directly. Rather, it is a common practice to infer foam texture indirectly from the pressure profile or apparent gas viscosity data. An estimation of foam texture in porous media cannot be made without having a proper understanding of the dynamic mechanisms of in-site lamella creation and coalescence.

\section{Foam Stability}

Foam stability can be quantified by the time required for collapsing half of the foam. Usually foam stability is tested by one of three methods (Schramm 2005, p47): 1) the lifetime of a single bubble; 2) the steady-state (dynamic) foam volume under given conditions of gas flow, shaking, or shearing; or 3) the rate of collapse of a (static) column of foam generated. The overall question of foam stability requires the consideration of both the static and dynamic aspects of bubble interactions. 
Rothmel et al. (1998) concluded that foam stability does not appear to be dependent upon inherent properties such as hydrophile-liphophile balance and CMC. Mulligan and Eftekhari (2003) evaluated the quality and stability of ten different commercial surfactants (Table 2.1), of which Triton X-100 and JBR425 showed better abilities to foam when compared with others. A series of experiments was performed by Wang and Mulligan (2004) to evaluate the foamability of rhamnolipid solutions with different concentrations $(0.5 \%, 1.0 \%$, and $1.5 \%)$. Results showed that foams could be generated from a low concentration of rhamnolipid solution ( $0.5 \%$ or perhaps less) with enough stability for injection. It was observed that foam quality and surfactant concentration had significant influences on foam stability. Generally, foam stability increased with the increase of surfactant concentration (up to 1.5\%), and the highest foam stability was produced with the quality between $90 \%$ and $99 \%$.

Table 2.1. Foamability Comparison of Different Surfactants (from Mulligan and Eftekhari 2003)

\begin{tabular}{|l|c|c|}
\hline \multicolumn{1}{|c|}{ Surfactants } & Stability (min) & Quality (\%) \\
\hline CHAPS & Negligible & Negligible \\
\hline Polytergen 305 LP & Negligible & Negligible \\
\hline Surfynol 104 A & Negligible & Negligible \\
\hline Triyon & Negligible & Negligible \\
\hline Taurodeoxycholic acid & Negligible & Negligible \\
\hline Triton X-100 & 6.2 & 99 \\
\hline Cetylpyridinium chloride & 6.2 & 95 \\
\hline JBR425 & 6.1 & 99 \\
\hline Igepal CA-630 & 4.6 & 98 \\
\hline Pluronic F68 & 6.1 & 95 \\
\hline
\end{tabular}

Foam density

The foam density can be calculated by

$$
\rho_{f}=\frac{m_{l}+m_{g}}{V_{f}}
$$

where $\mathrm{ml}$ and $\mathrm{mg}$ are the mass of liquid and gas, respectively, in the foam, and $\mathrm{Vf}$ is the total volume of the foam. In calculating the density of high-quality foam, the mass of gas involved can usually be ignored. Aqueous foam with bubble diameters of about $1 \mathrm{~cm}$ and lamellar thickness of 10-3 cm has a density of approximately $0.003 \mathrm{~g} \mathrm{~cm}-3$ (Myers 1999). For foams made from a liquid of density $\mathrm{\rho l}$ and volume Vl, the expansion factor (or ratio) of a foam, Rf, is defined as:

$$
R_{f}=\frac{\rho_{l}}{\rho_{f}}=\frac{V_{f}}{V_{l}}
$$




\section{Electrical Properties}

For Bulk foams, the specific conductivity is proportional to the volume fraction of liquid in the foam (Bikerman 1973):

$$
\frac{\kappa_{l}}{\kappa_{f}}=\frac{b \rho_{l}}{\rho_{f}}
$$

where $\mathrm{b}$ is the proportionality constant typically varying from 2.0 to 2.5 . However, $\mathrm{b}$ is also a function of $\rho_{\mathrm{l}} / \rho_{\mathrm{f}}$ and has the limit of 1 when $\rho_{\mathrm{l}} / \rho_{\mathrm{f}}=1$.

\subsection{Surface Tension of Surfactant Solution}

In an aqueous solution, surfactant at low concentration acts like normal electrolytes but has very different behavior results at high concentrations (Schramm 2005). The physico-chemical properties of surfactants vary sharply above and below the CMC value. A diagram of the surface tension variation with surfactant concentration is shown in Figure 2.1. According to Wang and Mulligan (2004) and Mulligan et al. (2001a), biosurfactants have the advantages over synthetic surfactants of lower toxicity, higher biodegradability, and better environmental compatibility. The CMCs of biosurfactants range from 1 to $2000 \mathrm{mg} \mathrm{L}^{-1}$. Surface tension and the interfacial tension of good biosurfactants are less than 30 and $1 \mathrm{mN}$

$\mathrm{m}^{-1}$, respectively (Mulligan and Gibbs 1993). Many surfactants can reduce the surface tension of water to approximately 25 $\pm 5 \mathrm{mN} \mathrm{m}^{-1}$ (Myers 1999; Wang and Mulligan 2004). Hence, the use of a surfactant can enhance the mobilization of connate soil water/solution in the vadose zone by reducing surface tension.

The Langmuir-von Szyszkowski equation is commonly used to estimate the interfacial tension (Aldana 2005):

$$
\sigma=\sigma_{0}-\Gamma_{\infty} R T \ln \left(1+\frac{C}{a_{L}}\right)
$$

where $\sigma$ is surface tension, $\sigma_{0}$ is the surface tension without surfactant, $C$ is surfactant concentration, $\Gamma_{\infty}$ is the maximum surface excess concentration, and $a_{\mathrm{L}}$ is the Langmuir constant. Both $\Gamma_{\infty}$ and $\mathrm{a}_{\mathrm{L}}$ are determined experimentally. Aldana (2005) measured the equilibrium surface tension vs. surfactant concentration for three surfactants (Figure 2.2). The results above CMC were described with Eq. [2.5], and the model parameters are summarized in Table 2.2. 


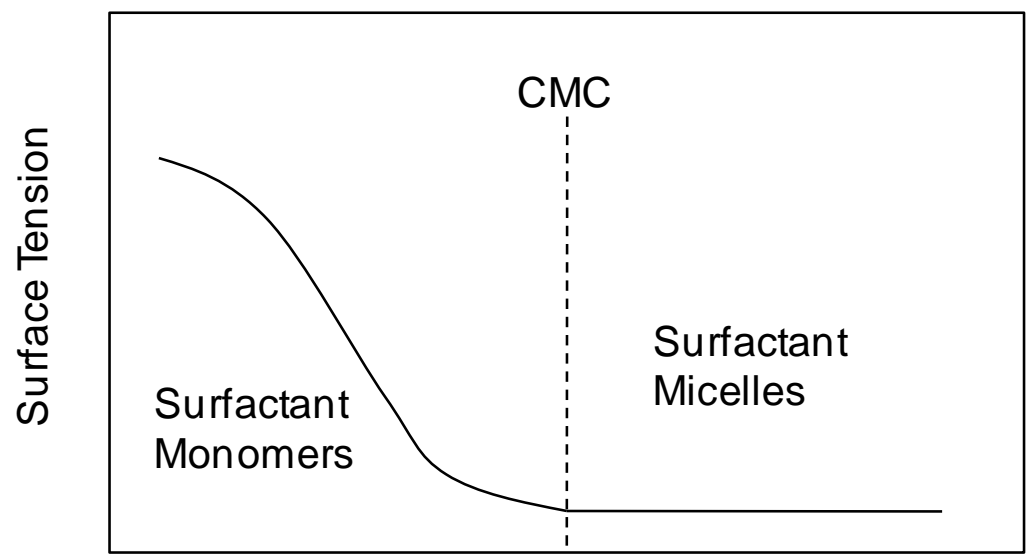

Log Concentration

Figure 2.1. Diagram of the Surface Tension Variation with the Surfactant Concentration (Drawn after Fig. 5 of Schramm and Wassmuth 1994). CMC - Critical micelles concentration.

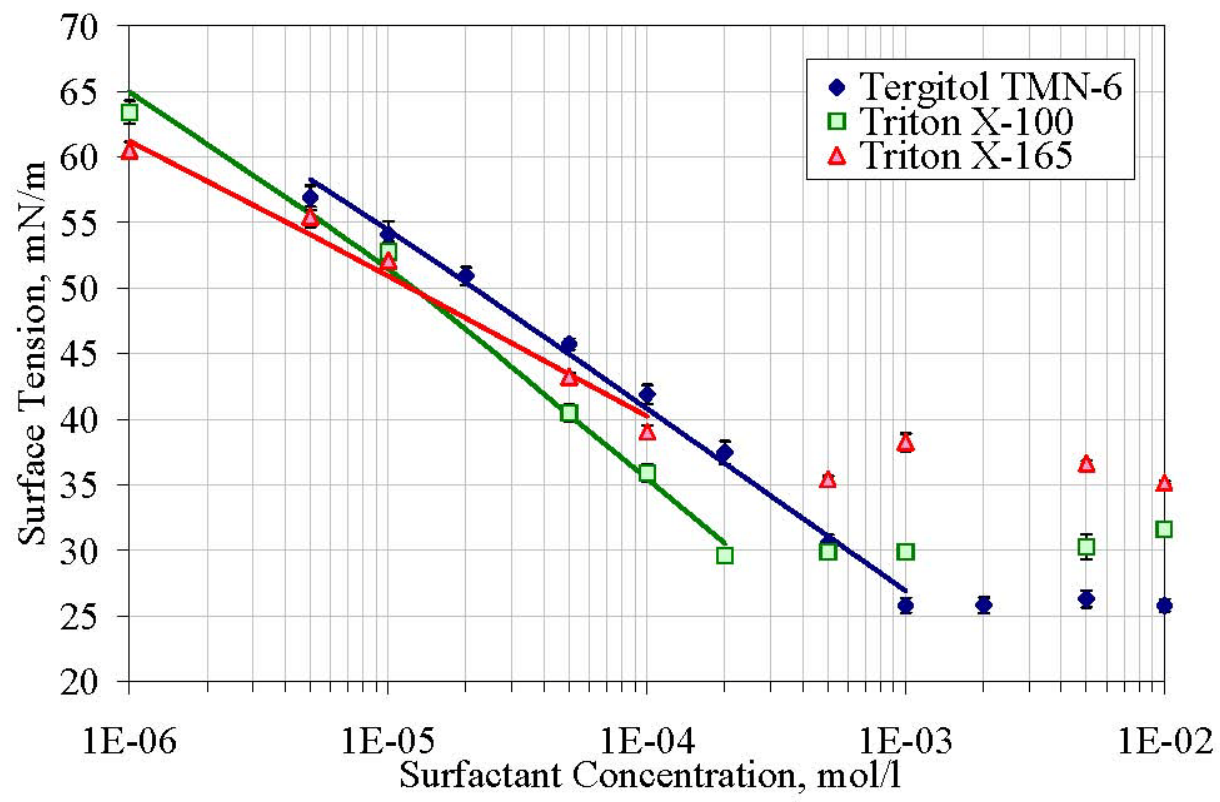

Figure 2.2. Measured Equilibrium Surface Tensions for Three Surfactants at $25^{\circ} \mathrm{C}$. Solid lines are the best fit to the Langmuir-von Szyszkowski equation, Eq. [2.5] (after Aldana 2005, Fig. 6.1.1-1). 
Table 2.2. Critical Micelle Concentration, Saturation Surface Excess Concentration $\left(\Gamma_{\infty}\right)$, and Langmuir Constant for Surfactants at the Water/Air Interface at $25^{\circ} \mathrm{C}$ (after Aldana 2005,

Table 6.1.1-1)

\begin{tabular}{|l|l|l|l|}
\hline & CMC $(\mathrm{mol} / \mathrm{L})$ & $\Gamma_{\infty}(\mathrm{mol} / \mathrm{m} 2)$ & $\mathrm{a}_{\mathrm{L}}(\mathrm{mol} / \mathrm{L})$ \\
\hline Tergitol TMN-6 & $1.03 \times 10-3$ & $2.44 \times 10-6$ & $5.77 \times 10-6$ \\
\hline Triton X-100 & $2.19 \times 10-4$ & $2.87 \times 10-6$ & $5.91 \times 10-6$ \\
\hline Triton X-165 & $1.44 \times 10-4$ & $1.88 \times 10-6$ & $1.09 \times 10-7$ \\
\hline
\end{tabular}

\subsection{Foam Disjoining Pressure}

The stability of a thin-liquid lamella is expressed by its disjoining pressure $\mathrm{P}_{\mathrm{d}}$ (Derjaguin and Kussakov 1939; Aronson et al. 1994). An idealized disjoining pressure isotherm is shown in Figure 2.3 for a film stabilized by an ionic surfactant (Kovscek and Radke 1994). Positive values of $P_{d}$ reflect net repulsive film forces while negative values of $\mathrm{P}_{\mathrm{d}}$ indicate net attractive forces. The disjoining pressure is composed of three components: electrostatic repulsion $\mathrm{P}_{\mathrm{d}}{ }^{\mathrm{el}}$, van der Waals attraction $\mathrm{P}_{\mathrm{d}}{ }^{\mathrm{vw}}$, and steric/hydration forces $\mathrm{P}_{\mathrm{d}}^{\text {sh }}$.

As the film thickness decreases from infinity, a primary maximum of $\mathrm{P}_{\mathrm{d}}$ occurs due to the repulsive electrostatic force overpowering the van der Waals attraction. Films at this thickness are referred to as common black films (CBF) or simply common thin films. As the thickness decreases further, the van der Waals attraction increases, and the slope of the isotherm varies. A primary minimum of $P_{d}$ is encountered at the point where short-distance steric/hydration forces become significant relative to the attraction force. As the film thickness decreases past the value corresponding to the minimum, the steric/hydration forces initiate a very steep repulsive branch. Films on this innermost branch are called Newton black films (NBF).

At a given capillary pressure, foam films thin or thicken to achieve an equilibrium thickness in accordance with the Young-Laplace relation:

$$
P_{c}=P_{d}(h)+2 C_{m} \sigma
$$

where $\mathrm{h}$ is film thickness, $\mathrm{C}_{\mathrm{m}}$ is the mean curvature of a lamella, and $\sigma$ is the bulk surface tension of the surfactant solution. As an approximation, the lamellae in porous media may be considered as flat (Aronson et al. 1994). For flat films $\left(C_{m}=0\right)$ at metastable equilibrium, the disjoining pressure balances the imposed capillary pressure at a region of the negative slope (Aronson et al. 1994):

$$
P_{c}=P_{d}(h)
$$

Thus, the film can exist in two possible equilibrium states, a CBF and a NBF (Figure 2.3). At some higher capillary pressure, the NBF ruptures. 


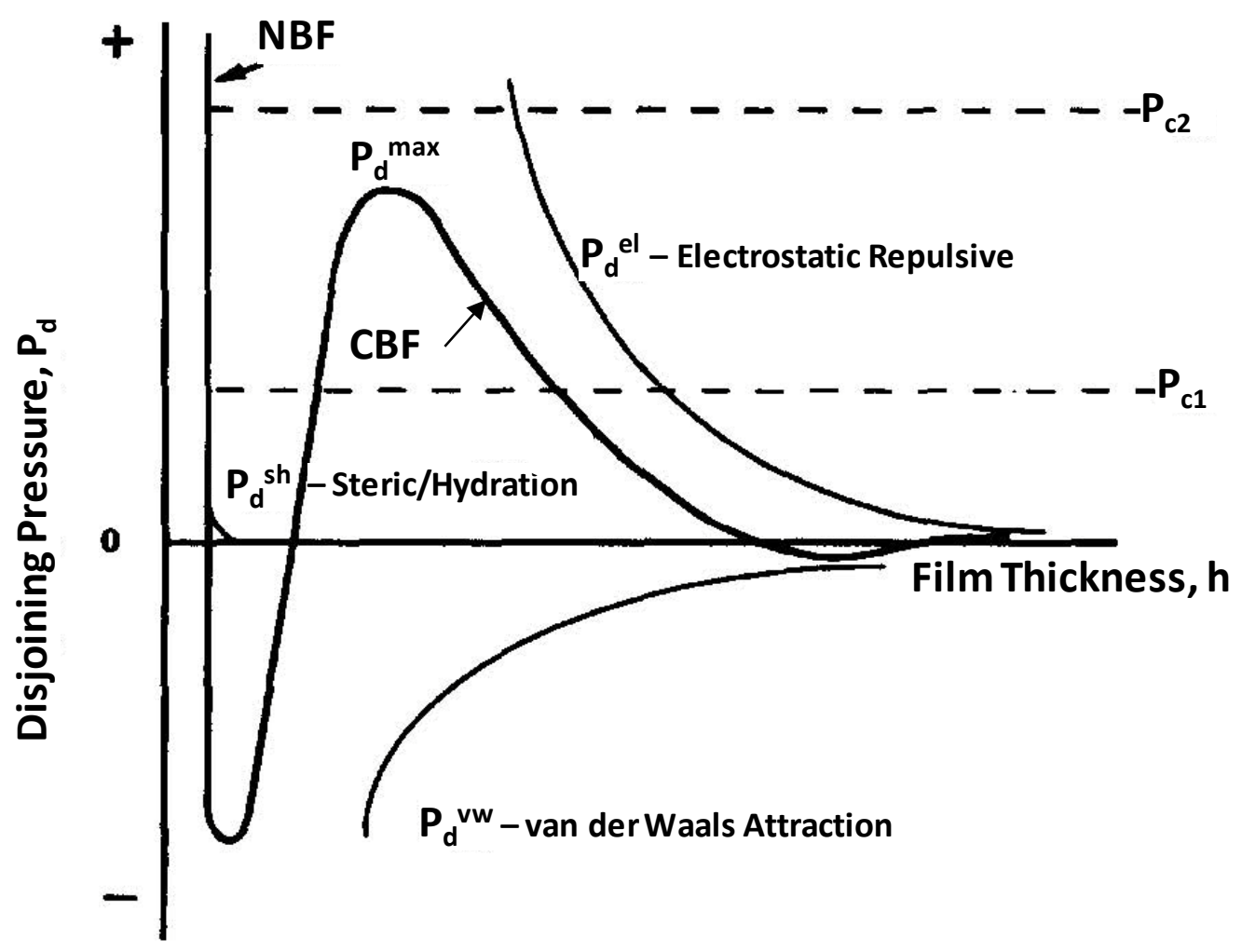

Figure 2.3. Idealized Disjoining Pressure Isotherm (Modified From Kovscek and Radke 1994). Definition of Variables: $P_{d}$ - disjoining pressure; $P_{d}{ }^{\text {el }}-P_{d}$ due to electrostatic repulsion; $P_{d}{ }^{v w}-$ $\mathrm{P}_{\mathrm{d}}$ due to the van der Waals attraction; $\mathrm{P}_{\mathrm{d}}^{\text {sh }}-\mathrm{P}_{\mathrm{d}}$ due to the steric/hydration forces; $\mathrm{P}_{\mathrm{c}}=$ capillary pressure; $\mathrm{P}_{\mathrm{c} 1}$ and $\mathrm{P}_{\mathrm{c} 2}=$ two $\mathrm{P}_{\mathrm{c}}$ values; $\mathrm{CBF}=$ common black film; $\mathrm{NBF}=$ Newton black film. 


\subsection{Vadose Zone Remediation with Foam}

In this section, experiments on foam transport in unsaturated porous media are reviewed. Compared with the work in the initially saturated media, a limited number of experiments have been performed in the initially unsaturated media.

\subsection{Remediation with Micro-Foam}

Micro-foam, or colloidal gas aphrons (CGAs), reportedly have been used for soil flushing in contaminated site remediation (Longe 1989; Roy et al. 1994, 1995a, b). CGAs consist of approximately $65 \%$ by volume gas and hence form a low-density liquid. The micro-bubbles have a double layer of surfactant molecules with a thin surfactant film encapsulating the air inside. CGAs offer a way of lowering the interfacial tension between organics and water while at the same time providing the viscous forces needed for efficient sweep of contaminants.

Longe (1989) reported that CGA suspensions were more effective in flushing a variety of hydrophobic organics from soil in comparison to conventional surfactant solutions at identical concentrations. Roy et al. (1994) compared the efficiency of CGA suspensions in the removal of oily waste with conventional surfactant solutions and water floods under different flow regimes. Soil was repacked in 11.5-cm-long, 5.9-cm-diameter columns and kept at residual saturation before experiments. Results show that CGA suspensions containing about $65 \%$ gas volume and produced using sodium dodecylsulfate had a higher recovery of waste material (56\%) than conventional surfactant solutions (47\%) or water flood (43\%) in the downflow (gravity-stable) mode. CGA suspensions appeared to provide better removal of the major chlorinated hydrocarbons present in the oily waste.

In a similar experiment to settings in Roy et al. (1994), Roy et al. (1995a) applied CGAs to flush residual levels of a light NAPL such as automatic transmission fluid (ATF) from a Superfund site soil. CGA suspensions were found to be more effective in washing ATF under both downflow and upflow modes. Increasing the surfactant concentration did not result in a concomitant increase in the removal rate. The pressure required to pump the CGA suspension was much lower than that required for conventional surfactant solutions or water flood. Roy et al. (1995b) used CGAs to remove naphthalene from a contaminated soil matrix in the laboratory. They found that using a CGA suspension as a flushing medium may result in channeling and pore clogging in the soil matrix, thereby affecting the overall efficiency of the process.

Rothmel et al. (1998) conducted a bench-scale study to evaluate the micro-foam remediation technology for mobilizing and dispersing dense non-aqueous phase liquid (DNAPL) combined with a bioaugmentation technology to remediate trichloroethylene (TCE) in situ. Silica quartz was packed in 10or 20-cm-long, 7.4-cm-internal-diameter columns and was kept saturated. Their results, using the anionic surfactant Steol CS-330, showed that the foam (of a 65\% to 75\% quality) injected into the TCE-DNAPLcontaminated sand columns enhanced mobilization of TCE-DNAPLs. Mobilization was maximized when the foam was injected in a pulsed operation. 


\subsection{Remediation with Polyhedral Foam}

Polyhedral foam differs from ball foam in that it has higher foam quality (higher fraction of gas) and lower density. The idea of using high-quality foam, in conjunction with air injection and vacuum, is to provide mobility control/containment of the treatment zone for a reduced groundwater contamination. A few laboratory column experiments have been conducted to investigate foam transport and the remediation of NAPL, metal or other contaminants using high-quality foam.

Kilbane et al. (1997) pointed out that the major obstacle to the implementation of the foam remediation technology was the high pressure required to move foam through soil. Pressure gradients of less than a critical value (usually 1 to $2 \mathrm{psi} / \mathrm{ft}$ depending on the depth of injection) are required to prevent soil heaving. They used columns of $30.4 \mathrm{~cm}$ in length and $7.6 \mathrm{~cm}$ diameter packed with 70 mesh sand to determine pressures needed to move aqueous and ethanol foams with foam quality from $97 \%$ to $99 \%$. They observed that pressure gradient decreased with increasing foam quality; however, even at the highest foam quality the lowest pressure drop observed was about $12 \mathrm{psi} / \mathrm{ft}$ for the aqueous foams. This indicates that highly stable foam cannot propagate through soil at the desired low pressures. They formulated IGT-FF52 surfactant to break and reform the foam bubbles constantly so that a balance between liquid and foam can be maintained. They observed that the ethanol-based foams formed from $5 \%$ (wt/v) IGT-FF52 exhibit pressure drops of 2 psi/ft or less over the full range of foam quality tested.

Kilbane et al. (1997) also illustrated how foams may be employed for subsurface remediation (Figure 3.1). They tested this concept in a 23-cm-high, 16.5-cm-diameter cylindrical vessel. The column was packed with alternating dry sand layers of different permeability to simulate hydraulic fractures (Figure 3.2). The top of the column was open to the atmosphere. A small-diameter plastic tube having a circular perforated loop at one end was designed to supply foam (or water) and spread it uniformly in the injection zone. A similar arrangement was utilized to supply air at $150 \mathrm{ml} / \mathrm{min}$ to the bottom fracture layer and to draw vacuum ( 2 in. of $\mathrm{Hg}$ ) from the top fraction layer. The same experiment was repeated in a bench-scale 38-cm-high, 29-cm-diameter column. The movement of the surfactant solution was compared with that of water using an identical experiment setup. They found that when water was injected, there was almost no upward flow despite the injection of $150-\mathrm{ml} / \mathrm{min}$ air into the lowest fracture plane and a vacuum applied to the upper fracture plane. In contrast, the IGT-FF52 solution moved at roughly equivalent rates in both the upward and downward directions. Foam coalescence was observed upon contacting the dry sand. The authors claimed that the upward movement of IGT-FF52 solution indicated it behaved as a foam that was being reformed from the collapsed foam by the action of air injection and vacuum so that the upward movement of the foam through the sand continued. 


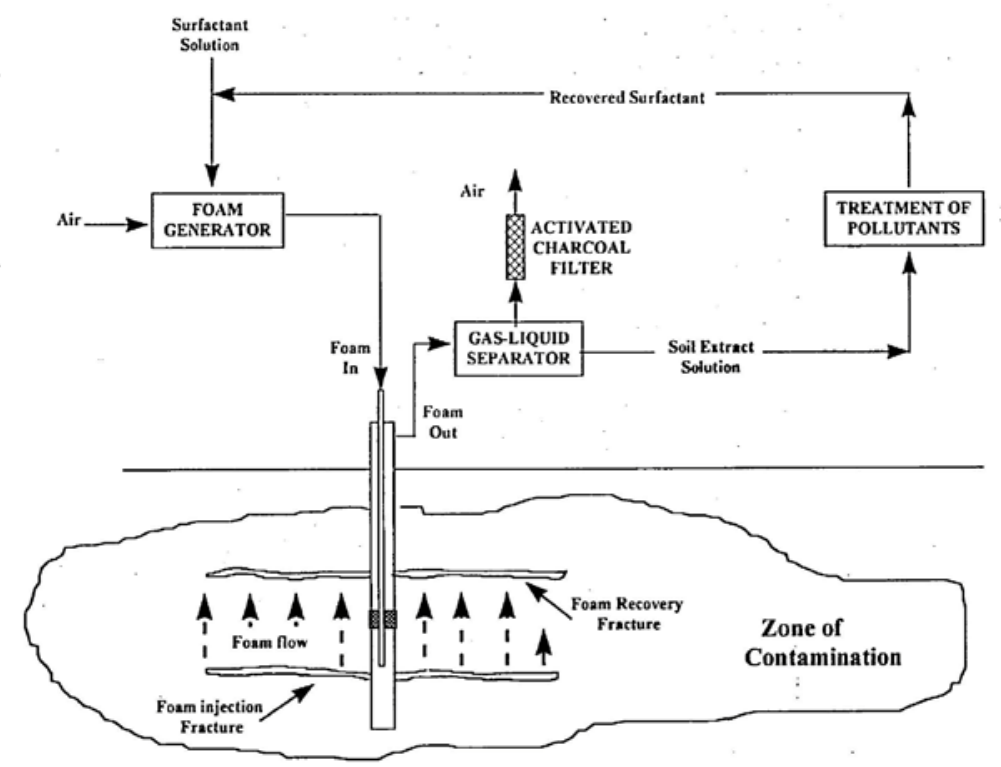

Figure 3.1. Schematic Illustration of the Use of Foams to Remediate Contaminated Soil (After Kilbane et al. 1997)

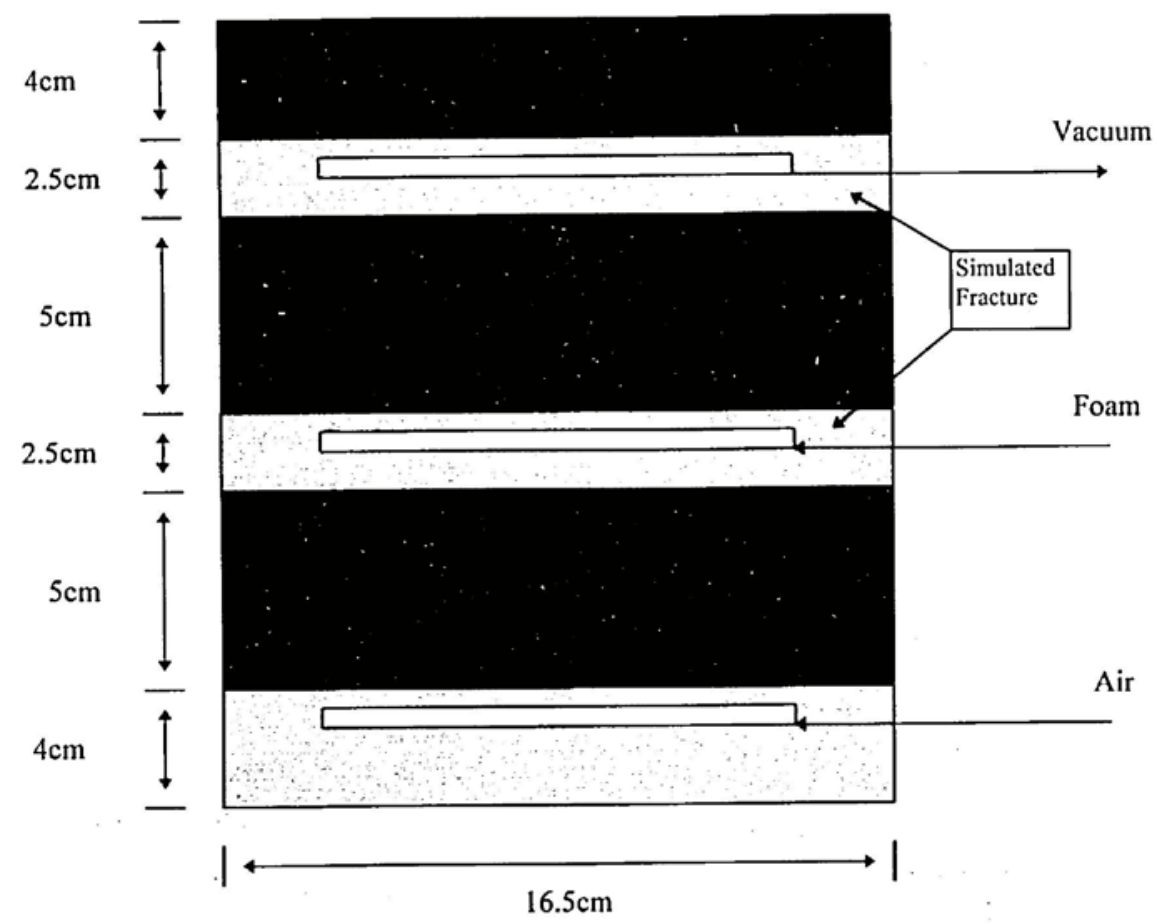

Figure 3.2. Schematic Drawing of Soil and Simulated Fracture Zones in a Cylindrical Bench-Scale Chamber Used for Foam Flow Tests (After Kilbane et al. 1997) 
Mulligan and Eftekhari (2003) evaluated the capability of foam for removing the contaminant pentachlorophenol (PCP) from soils. Several surfactants were investigated for their ability to make foam. Soils were packed in 15-cm-long, 3.5-cm-diameter columns. Two of the surfactant used, Triton X-100 and JBR425 (a rhamnolipid biosurfactant), generated foam with higher quality (99\%) and higher stability than the other surfactants. They found that the foam removed more than twice as much PCP in all cases than the liquid surfactant solution. The results on a sandy and sandy-silt media showed that the foam can be used to enhance soil remediation under low pressures compared to other fluids such as liquid surfactant solutions. Mulligan and Wang (2006) found that rhamnolipid foam may be an effective and non-toxic method of remediating heavy metal contaminated soils.

Surfactant foam technology has been investigated to remove NAPLs (Peters et al. 1994; Kilbane et al. 1997; Rothmel et al. 1998; Jeong et al. 2000) from contaminated soils. Foams enhance the flooding efficiency of surfactant flushing even in a heterogeneous porous medium, resulting in higher removal efficiencies (Jeong et al. 2000). A summary of work involving surfactant foam enhanced remediation was reported in Wang and Mulligan (2004) and is reproduced in Table 3.1. 
Table 3.1. Summary of Surfactant Foam Enhanced Soil Remediations (from Wang and Mulligan 2004)

\begin{tabular}{|c|c|c|c|}
\hline Description & Surfactants & Main Results & References \\
\hline $\begin{array}{l}\text { Filed demonstration of } \\
\text { surfactant/foam process for aquifer } \\
\text { NAPL (mixture of TCE, TCA }^{\mathrm{a}} \text { and } \\
\text { PCE }^{\mathrm{b}} \text { ) remediation at Hill Air Force } \\
\text { Base in Utah. }\end{array}$ & $\begin{array}{l}\text { Sodium } \\
\text { dihexylsulfosuc } \\
\text { cinate }\end{array}$ & $\begin{array}{l}\text { The average DNAPL saturation of } \\
\text { the swept volume was reduced to } \\
0.03 \%\end{array}$ & $\begin{array}{l}\text { Hirasaki et al. } \\
\text { (1997b) }\end{array}$ \\
\hline $\begin{array}{l}\text { Foam-enhanced surfactant solution } \\
\text { flooding in removing n-pentadecane } \\
\text { from contaminated column }\end{array}$ & Triton SP-series & $\begin{array}{l}\text { Slightly over } 74 \% \text { of the } n- \\
\text { pentadecane was removed at a } \\
\text { gas-liquid ratio of } 10 / 1\end{array}$ & $\begin{array}{l}\text { Huang and } \\
\text { Chang (2000) }\end{array}$ \\
\hline $\begin{array}{l}\text { Micromodel study of surfactant } \\
\text { foam remediation of residual TCE }\end{array}$ & Bioterge As-40 & $\begin{array}{l}99 \% \text { of the residual TCE was } \\
\text { removed }\end{array}$ & $\begin{array}{l}\text { Jeong et al. } \\
\text { (2000) }\end{array}$ \\
\hline $\begin{array}{l}\text { Remediation of } \mathrm{PAH}^{\mathrm{C}} \text {-contaminated } \\
\text { soils using foams }\end{array}$ & $\begin{array}{l}\text { Biosurfactants } \\
+50 \% \text { ethanol }\end{array}$ & $\begin{array}{l}\text { Foams readily desorbed PAHs } \\
\text { from contaminated soils and } \\
\text { moved well at pressure of } \\
33.9 \mathrm{kPa} / \mathrm{m}(1.5 \mathrm{psi} / \mathrm{ft}) \text { or less }\end{array}$ & $\begin{array}{l}\text { Kilbane et al. } \\
\text { (1997) }\end{array}$ \\
\hline $\begin{array}{l}\text { Soil flushing using CGA } \\
\text { suspensions generated from a plant- } \\
\text { based surfactant to remove } \mathrm{HCB}^{\mathrm{e}} \\
\text { from soil }\end{array}$ & $\begin{array}{l}\text { Natural } \\
\text { surfactant from } \\
\text { Sapindus } \\
\text { mukorossi } \\
\text { (Soapnut) }\end{array}$ & $\begin{array}{l}\text { CGA suspension recovered } 670 \mathrm{lg} \\
\text { in } 12 \text { pore volumes compared to } \\
8 \mathrm{l} \mathrm{g} \text { by water flood }\end{array}$ & $\begin{array}{l}\text { Kommalapati } \\
\text { et al. (1998) }\end{array}$ \\
\hline $\begin{array}{l}\text { Remediation with surfactant foam } \\
\text { of PCP } \text {-contaminated soil }^{\mathrm{e}}\end{array}$ & $\begin{array}{l}\text { Triton X-100, } \\
\text { JBR } 425\end{array}$ & $\begin{array}{l}\text { Triton X-100 (1\%) foam removed } \\
85 \% \text { and } 84 \% \text { of PCP from fine } \\
\text { sand and sandy-silt, respectively }\end{array}$ & $\begin{array}{l}\text { Mulligan and } \\
\text { Eftekhari (2003) }\end{array}$ \\
\hline $\begin{array}{l}\text { Bench-scale study of surfactant } \\
\text { foam/ bioaugmentation technology } \\
\text { for in situ treatment of TCE- } \\
\text { DNAPLs }\end{array}$ & Steol CS-330 & $\begin{array}{l}\text { Injecting the foam in a pulsed } \\
\text { operation removed } 75 \% \text { of the } \\
\text { contaminant, and adding the } \\
\text { microbes resulted in 95-99\% } \\
\text { degradation of the residual }\end{array}$ & $\begin{array}{l}\text { Rothmel et al. } \\
\text { (1998) }\end{array}$ \\
\hline $\begin{array}{l}\text { Column tests to evaluate } \\
\text { rhamnolipid foam-enhanced } \\
\text { remediation of Cd and } \mathrm{Ni} \\
\text { contaminated soil }\end{array}$ & $\begin{array}{l}\text { JBR 425, Triton } \\
\text { X-100 }\end{array}$ & $\begin{array}{l}\text { Foam removed } 73 \% \text { of the } \mathrm{Cd} \text { and } \\
68 \% \text { of the Ni from the } \\
\text { contaminated soil }\end{array}$ & $\begin{array}{l}\text { Wang and } \\
\text { Mulligan (2004) }\end{array}$ \\
\hline
\end{tabular}





\subsection{Foam Transport Processes in Porous Media}

This section reviews the transport processes of foam in porous media followed by the characteristic of foam flow and some mathematical expressions.

\subsection{Foam Dynamic Processes}

Major foam dynamic processes include foam destruction, formation, and entrapment. Processes of surfactant sorption/desorption are not considered here.

\subsubsection{Foam Destruction}

Thin lamellae are not thermodynamically stable. They owe their existence to excess normal forces within the films originating from long-range intermolecular interactions (see Section 2.3). Adsorption of ionic surfactant at each gas/liquid surface of the film gives rise to the excess repulsive forces. At small film thicknesses, protrusion and/or hydration forces give rise to a very steep repulsion. Additionally, attractive van der Waals forces tend to destabilize the film. These combined three forces form the disjoining pressure $P_{d}$, which is a function of film thickness. $P_{d}$ reaches its maximum value, $P_{d}{ }^{\text {max }}$, as a 4-nm-thick NBF emerges. As $P_{d}$ reaches a value $P_{d}{ }^{\text {rup }}$, the film eventually ruptures. For bulk foam systems, the $P_{d}^{\text {rup }}$ is a well-documented parameter controlling the stability of the foam (Khristov et al. 1981).

The primary mechanism for foam destruction in porous media is due to capillary suction $\mathrm{P}_{\mathrm{c}}$ (Kovscek and Radke 1994). The capillary pressure depends on the wetting liquid saturation and surface tension. The $P_{c}$ value corresponding to $\mathrm{P}_{\mathrm{d}}{ }^{\text {rup }}$ is termed the critical capillary pressure for rupture, $\mathrm{P}_{\mathrm{c}}{ }^{*}$. As $\mathrm{P}_{\mathrm{c}}{ }^{*}$ is reached, no foam bubbles can be sustained, and coalescence is catastrophic. Since capillary pressure is related to water saturation in porous media, there is a water saturation that corresponds to $\mathrm{P}_{\mathrm{c}}{ }^{*}$ called $\mathrm{S}_{\mathrm{w}}{ }^{*}$. The expression describing the foam destruction process is presented in Section 5.3.1.1.

\subsubsection{Foam Formation}

There are three fundamental pore-level mechanisms of foam generation: snap-off, division, and leavebehind. The snap-off mechanism is a mechanical process that describes the formation of bubbles when the gas pushes the gas-liquid interface through a pore throat and then the interface is snapped off. Snap-off creates gas bubbles that are approximately the size of the pores of the porous medium. Snap-off usually creates discontinuous gas foam and can result in a several-hundred-fold reduction in gas mobility. The division of lamella or bubble division happens by subdividing existing foam bubbles or lamellae. The leave-behind mechanism describes the formation of liquid lenses left behind as two gas menisci invade the adjacent liquid-filled pore bodies and converge downstream. Lenses created by leave-behind do not make the gas phase discontinuous. The expression describing the foam formation process is presented in Section 5.3.1.1.

\subsubsection{Foam Entrapment}

In general, the saturation of trapped foam, $\mathrm{s}_{\mathrm{ft}}$, is a function of pressure gradient, capillary pressure, aqueous-phase saturation, pore geometry, and injection conditions. Generally, there is no trapping when 
the bubble density is zero and a gradual rise to a maximum trapping for finer-textured foam. The trapped foam strongly influences the foam relative permeability by reducing the mobile foam saturation.

The trapped foam fraction usually is measured experimentally at steady state (Friedmann et al. 1991). However, percolation models may be able to determine the functional dependence of $\mathrm{s}_{\mathrm{ft}}$ (Rossen 1990a, b, c, d; Rossen and Gauglitz 1990). The relationship between the trapped foam saturation and foam texture is presented in Section 5.3.1.2.

\subsection{Foam Flow}

The behavior of foam in porous media is related to the pore-size distribution, pore shape, pore body-tothroat ratio, and the wettability of the solid particles. Most natural soils are hydrophilic, and hence, the wetting fluid (e.g., water) flows in interconnected small pore channels, the corners, and thin films around solid particles, while the non-wetting fluid (e.g., gas) flows in the interconnected large pore channels. The transport of foam in porous media is complicated in that the number of lamellae present governs flow characteristics such viscosity, relative permeability, fluid distribution, and interactions between fluids.

\subsubsection{Regimes of Foam Flow}

Coarsely textured foam with large bubbles is often referred to as "weak foam" because there is a moderate reduction in gas mobility. Vice versa, finely textured foam with small bubbles is referred to as "strong foam" because it reduces gas mobility remarkably. Figure 4.1 schematically shows the two different types of foams in porous media. Reduced gas mobility in the presence of foam in porous media typically ranges from 10 to 100 times with weak foam and more than 10,000 times with strong foam. In other words, the rheological property of foam in porous media is strongly affected by foam texture.

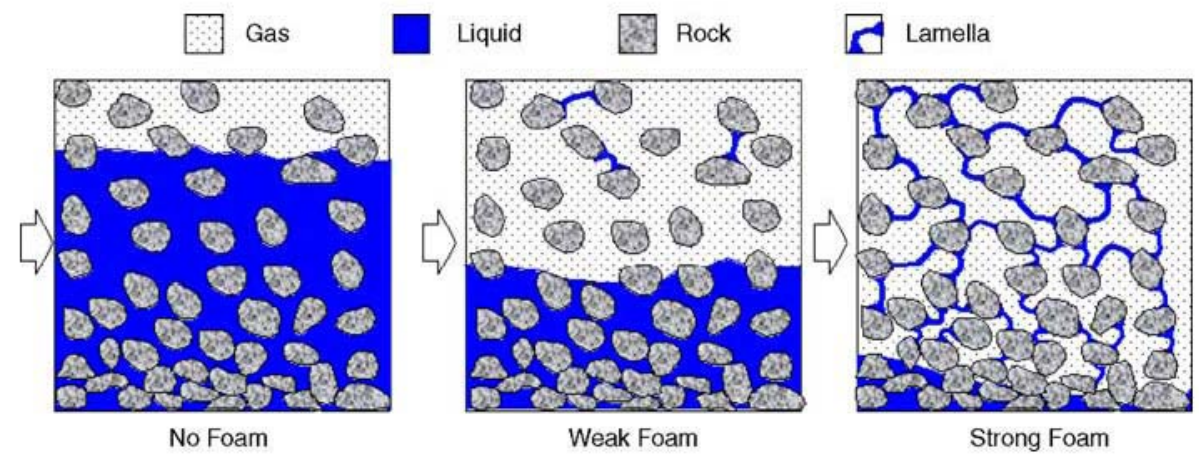

Figure 4.1. Concept of the Gas-Water, Two-Phase Flow and Weak and Strong Foams in Porous Media (from Fig. 1 of Dholkawala et al. 2007)

There is an abrupt transition from strong foam to weak foam near the limiting or critical capillary pressure, $\mathrm{P}_{\mathrm{c}}{ }_{\mathrm{c}}$, above which lamellae cannot be sustained. There is a specific value of foam quality, $\mathrm{f}^{*}$, that divides the foam flow into the two regimes. At the low-quality regime, the steady-state pressure gradient is almost independent of the liquid flow rate. At the high-quality regime, the pressure gradient is almost independent of the gas flow rate. Previous studies found that the two regimes are dominated by different mechanisms: the high-quality regime by bubble coalescence near the critical capillary pressure and the low-quality regime by bubble trapping and mobilization (Khatib et al. 1988; Rossen and Wang 1999; 
Alvarez et al. 2001). The bubble size in the low-quality regime is generally kept roughly the same as the pore size, and thus foam texture $\left(\mathrm{n}_{\mathrm{f}}\right)$ is almost fixed at its maximum $\left(\mathrm{n}_{\mathrm{f}}{ }^{\max }\right)$. Gauglitz et al. (2002), through experiments, found that an intermediate unstable regime exists between the weak and strong foam regimes.

\subsubsection{Minimum Pressure Gradient}

A minimum pressure gradient, $\mathrm{G}_{\mathrm{p}}{ }^{\mathrm{min}}$, is required to keep foam moving in a porous medium. If the pressure gradient is not sufficient to keep the lamellae mobile, gas flow stops, and the foam plugs the flow channels. Rossen (1990a) presented a theory to accounts for pore shape, foam texture, contact-angle hysteresis, and bubble separation at pore throats. The magnitude of $G_{p}{ }^{\min }$ is dependent on pore shape, foam texture, and surface tension. For medium-textured foams (bubbles of about $400 \mu \mathrm{m}$ in diameter in bulk), reasonable parameter values give $\mathrm{G}_{\mathrm{p}}{ }^{\mathrm{min}}=10$ to $32 \mathrm{kPa} / \mathrm{m}$ for $\mathrm{CO}_{2}$ foam and 125 to $240 \mathrm{kPa} / \mathrm{m}$ for other foam. Lower gas/liquid surface tension causes the lower $\mathrm{G}_{\mathrm{p}}{ }^{\min }$ estimate for $\mathrm{CO}_{2}$ foams. Higher values of $G_{p}{ }^{m i n}$ are predicted for foams in pores with larger aspect ratio (body radius/throat radius), sharp corners, narrow throats, and at lower capillary pressures. $G_{p}{ }^{m i n}$ is reduced by lower gas/liquid surface tension but increased if foam is compressed because, in compressible foams, lamellae tend to lodge in pore throats, where capillary resistance to flow is the greatest (Rossen 1990b).

\subsubsection{Darcy's Law}

The movement of flowing foam is described by Darcy's law (Kovscek and Radke 1994):

$$
\begin{aligned}
q_{f} & =\frac{k k_{r}}{\mu_{f}}\left[-\frac{\partial p_{g}}{\partial x}\right] \\
& =K\left[-\frac{\partial p_{g}}{\rho_{w} g \partial x}\right]
\end{aligned}
$$

where $\mathrm{k}$ is the absolute permeability, $\mathrm{k}_{\mathrm{rf}}$ is the relative permeability to the flowing foam, $\mu_{\mathrm{f}}$ is the foam effective viscosity, $\mathrm{K}$ is effective mobility, $\rho_{\mathrm{w}}$ is the density of water, and $\mathrm{g}$ is gravity. Equation [4.1] does not imply Darcy flow because $\mu_{\mathrm{f}}$ is not a constant. For the same reason, $\mathrm{K}$ also varies as the foam gradient varies.

\subsubsection{Limiting Capillary Pressure}

Capillary pressure $\left(\mathrm{P}_{\mathrm{c}}\right)$ governs foam texture. The higher the capillary pressure, the less stable are the foam lamella. In sand- and bead-pack experiments, Kahtib et al. (1988) identified an abrupt transition from a strong foam to no foam (or weak foam) at a value of capillary pressure, $\mathrm{P}_{\mathrm{c}}{ }^{*}$, which is called the "limiting capillary pressure.” Because gas mobility depends on foam texture, there is also an abrupt change in gas mobility in the immediate vicinity of $\mathrm{P}_{\mathrm{c}}{ }^{*}$. The nature of this change, and the magnitude of $\mathrm{P}_{\mathrm{c}}$ at which it occurs, depend on the surfactant type and concentration, soil type, and other variables.

Simulation of strong foam in the high-quality regime is challenging because changes in bubble size are very sensitive to injection conditions, such that the capillary pressure stays near $\mathrm{P}_{\mathrm{c}}{ }^{*}$ by a delicate feedback 
mechanism (Kam and Rossen 2003; Cheng et al. 2002; Rossen and Bruining 2007; Kam et al. 2007). According to Kibodeaux (1997) and Kam (2008), the feedback mechanism near $\mathrm{P}_{\mathrm{c}}{ }^{*}$ can be summarized as shown in Figure 4.2. Aronson et al. (1994) and Khatib et al. (1988) summarized how to measure $\mathrm{P}_{\mathrm{c}}{ }^{*}$ experimentally and the influencing factors.

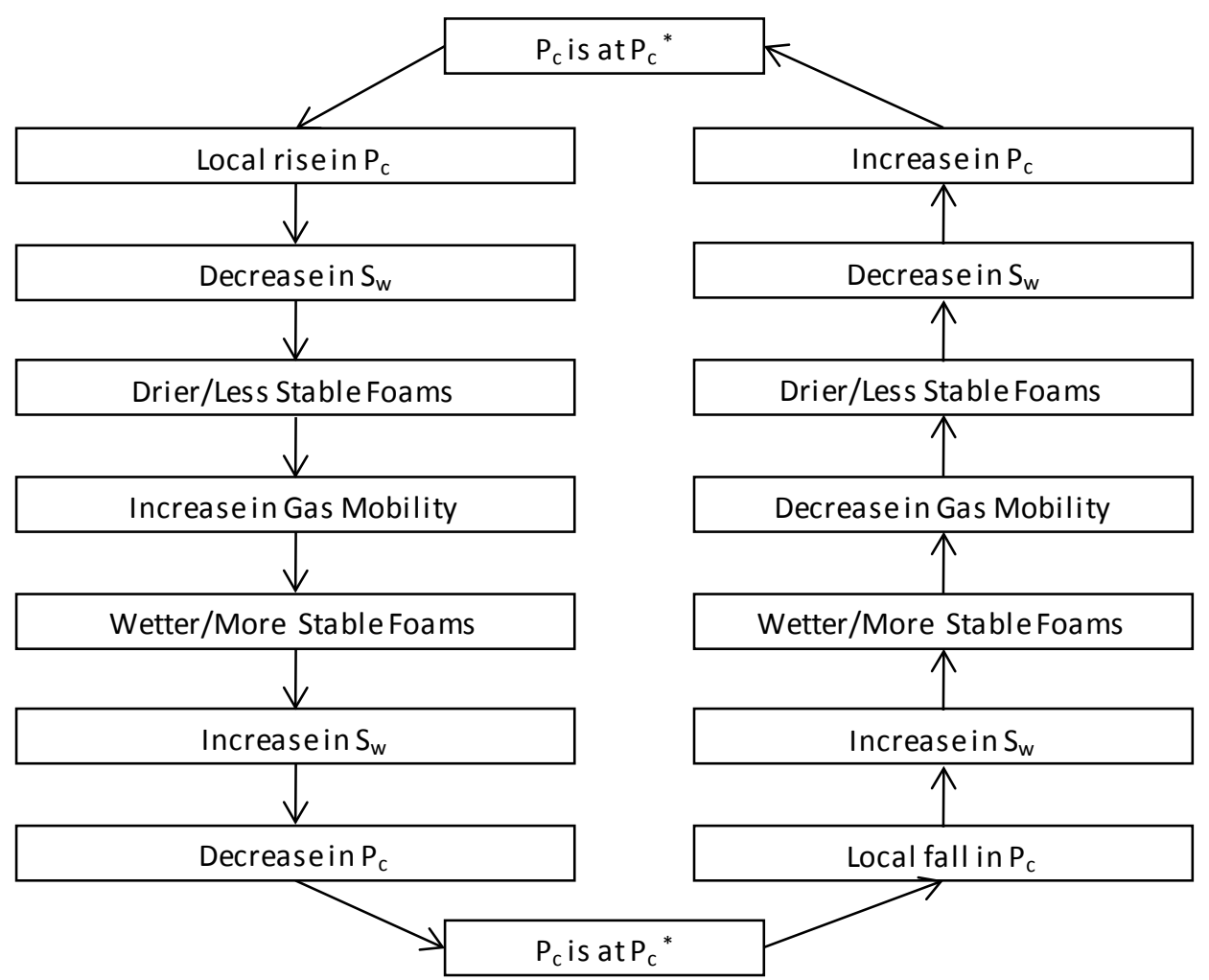

Figure 4.2. Feedback Mechanism Near the Limiting Capillary Pressure, $\mathrm{P}_{\mathrm{c}}$.

The abrupt change in gas mobility at $\mathrm{P}_{\mathrm{c}}{ }^{*}$ means that the system is maintained at $\mathrm{P}_{\mathrm{c}}{ }^{*}$ over a variety of water and gas flow rates. Because water saturation, $\mathrm{S}_{\mathrm{w}}$, depends on $\mathrm{P}_{\mathrm{c}}$, and the relative permeability function $\mathrm{k}_{\mathrm{rw}}\left(\mathrm{S}_{\mathrm{w}}\right)$ is unaffected by foam (Bernard et al. 1965), the pressure gradient, $\nabla \mathrm{P}$, can be determined simply from Darcy's law applied to the aqueous phase:

$$
\nabla P=\frac{q_{w} \mu_{w}}{k k_{r w}\left(S_{w}^{*}\right)}=\frac{q_{w}}{\lambda_{w}^{*}}
$$

where $\lambda_{\mathrm{w}}{ }^{*}$ is water mobility at saturation $\mathrm{S}_{\mathrm{w}}{ }^{*}=\mathrm{S}_{\mathrm{w}}\left(\mathrm{P}_{\mathrm{c}}{ }^{*}\right)$. In the absence of significant $\mathrm{P}_{\mathrm{c}}$ gradients, $\nabla \mathrm{P}$ is the same in the water and gas phases. Therefore, for foams at $\mathrm{P}_{\mathrm{c}}{ }^{*}, \nabla \mathrm{P}$ can be calculated without knowing foam texture, if the value of $\mathrm{k}_{\mathrm{rw}}\left(\mathrm{S}_{\mathrm{w}}{ }^{*}\right)$ is known (Khatib et al. 1988; Rossen and Zhou 1995). Eq. [4.2] represents an enormous simplification over other mechanistic foam-modeling approaches (e.g., bubble population and percolation theory). The determination of the individual lamella generation, trapping, and destruction mechanisms, as well as the determination of non-Newtonian gas mobility from foam texture 
and flow rates, are unnecessary for foams at their limiting capillary pressure. In essence, for foams at $\mathrm{P}_{\mathrm{c}}{ }_{\mathrm{*}}$, foam texture adjusts to the values is required to maintain $\mathrm{P}_{\mathrm{c}}$ at $\mathrm{P}_{\mathrm{c}}{ }^{*}$.

\subsubsection{Effective Viscosity of Foam}

Based on the theoretical studies of Bretherton (1961) and Hirasaki and Lawson (1985), the effective viscosity of the non-Newtonian foam is described as

$$
\mu_{f}=\mu_{g}+\frac{\alpha n_{f}}{v_{f}^{c}}
$$

where $\mu_{\mathrm{g}}$ is the viscosity of gas without foam, $\mathrm{v}_{\mathrm{f}}$ is the foam velocity, $\alpha$ is a proportionality constant dependent primarily on the surfactant solution, and c is also a constant. Other researchers have presented similar expressions (Ettinger 1989; Friedmann et al. 1991; Falls et al. 1989). According to Eq. [4.3], the effective foam viscosity increases with foam texture but decreases with foam velocity. In the absence of flowing foam bubbles (i.e., $\mathrm{n}_{\mathrm{f}}=0$ ), the gas viscosity is recovered. Friedmann et al. (1991) reported an empirical value of 0.29 for the exponent c. The Bretherton-based theoretical value is $1 / 3$ (Bretherton 1961; Hirasaki and Lawson 1985; Falls et al. 1989).

Falls et al. (1989) developed a different theoretical model to account for the pore constriction in homogeneous bead packed in glass tubes. Four components contribute to the effective viscosity $\mu \mathrm{f}: 1) \mu_{1}$, the liquid viscosity of the slugs of surfactant solution between the bubbles, 2) $\mu_{2}$, the resistance between bubbles and channel walls during foam flow, 3) $\mu_{3}$, the surface-tension gradient due to the surfactant concentration gradient, and 4) $\mu_{4}$, the resistance due to pore constrictions. Each component is expressed by (Jeong and Corapcioglu 2003) as

$$
\begin{gathered}
\mu_{1}=\mu_{w} L_{s} n_{L} \\
\mu_{2}=\mu_{w}\left[\frac{0.85 n_{L} r_{c a p}}{r_{c} / r_{c a p}}\right]\left(3 \mu_{w} u_{g} / \sigma\right)^{-1 / 3}\left[1+\left(r_{c} / r_{\text {cap }}\right)^{2}\right] \\
\mu_{3}=\mu_{w} n_{L} r_{\text {cap }}\left(3 \mu_{w} u_{g}\right)^{-1 / 3} \sqrt{N_{s} \tanh \left(L_{B D} / 2\right)} \\
\mu_{4}=\mu_{w} \xi_{1} n_{L} r_{c a p}\left(3 \mu_{w} u_{g} / \sigma\right)^{-1}
\end{gathered}
$$

where

$\mu_{\mathrm{w}}$ - viscosity of the aqueous phase

$\mathrm{L}_{\mathrm{s}}$ - length of liquid slugs between the bubbles

$\mathrm{n}_{\mathrm{L}}$ - number of lamellae per unit length

$\mathrm{r}_{\text {cap }}-$ capillary radius, $\mathrm{r}_{\text {cap }}=\phi \mathrm{d}_{\mathrm{b}} /\left[3(1-\phi)+2 \mathrm{~d}_{\mathrm{b}} / \mathrm{d}_{\mathrm{t}}\right], \phi$ - porosity, $\mathrm{d}_{\mathrm{b}}$ - bead diameter, $\mathrm{d}_{\mathrm{t}}-$ tube diameter

$r_{c}$ - radius of curvature of plateau border in foam lamella, $r_{c}=\left\{(1-f) /[3(1-\pi / 4) f]\left(r_{B} / r_{\text {cap }}\right)^{3}\right\}^{1 / 2} r_{\text {cap }}, f-$ foam quality, $\mathrm{r}_{\mathrm{B}}$ - foam bubble radius

$N_{s}$ - dimensionless bubble group, $N_{s}=\beta / r_{c} \cdot \beta=5 \mathrm{~cm}$ is the parameter in the smooth-tube viscosity model

$\mathrm{L}_{\mathrm{BD}}$ - dimensionless bubble length, $\mathrm{L}_{\mathrm{BD}}=\left(\mathrm{L}_{\mathrm{B}} / \mathrm{r}_{\mathrm{c}}\right)\left(3 \mu_{\mathrm{w}} \mathrm{u}_{\mathrm{g}} / \sigma\right)^{-1 / 3} / \mathrm{N}_{\mathrm{s}}^{1 / 2}, \mathrm{~L}_{\mathrm{B}}-$ length of bubbles

$\xi_{1}$ - geometrical factor. 
The total of the effective foam viscosity is the sum of the four components, i.e., $\mu_{\mathrm{f}}=\mu_{1}+\mu_{2}+\mu_{3}+\mu_{4}$. Jeong and Corapcioglu (2003) calculated each of the four components and found that $\mu_{2}$ and $\mu_{4}$ were the dominant contributors to the total effective viscosity.

Chowdiah et al. (1998) experimentally determined the effective viscosity of an ethanol-based foam as a function of soil permeability. They found that the effective viscosity of the foam increased with increasing soil permeability. In other words, this foam behaved like a more viscous fluid in highpermeability soils than in low-permeability soils. They also found that the mobility of a foam, defined by the ratio of soil permeability to effective viscosity, varied very little over a wide range of soil permeabilities. The characteristic that foam mobility is relatively independent of soil permeability is useful in preventing preferential flow in high permeability channels.

\subsubsection{Effective Mobility of Foam}

Effective mobility contains the combined effects of foam permeability and effective viscosity (Eq. [4.1]). The effective mobility of foam may be determined with effective viscosity or measured experimentally using Eq. [4.1]. Mulligan and Wang (2006) measured the pressure drop across a 25-cm-long, 4-cmdiameter column containing a soil of hydraulic conductivity of $0.02 \mathrm{~cm} / \mathrm{s}$. Their experimental results showed that the pressure gradient buildup in the soil column increased as foam quality decreased from $99 \%$ to $90 \%$. Increasing the flow rates from $10 \mathrm{ml} / \mathrm{min}$ to $30 \mathrm{ml} / \mathrm{min}$ also increased the pressure gradient in an almost linear fashion. The average hydraulic conductivities were $4.1 \times 10^{-4} \mathrm{~cm} / \mathrm{s}, 1.5 \times 10^{-4} \mathrm{~cm} / \mathrm{s}$ and $2.9 \times 10^{-3} \mathrm{~cm} / \mathrm{s}$ for the $90 \%, 95 \%$, and $99 \%$ foam qualities, respectively. They were about $2 \%, 0.8 \%$, and $15 \%$ of the hydraulic conductivity for water. Increasing the foam quality substantially increased the hydraulic conductivity. 


\subsection{Modeling Foam Transport}

Three major approaches to modeling foam transport in porous media are the empirical, semi-empirical, and mechanistic. Mechanistic approaches can be complete in principle, but may be difficult for obtaining reliable parameters, whereas empirical and semi-empirical approaches can be limited by the detail used to describe foam rheology and mobility. Mechanistic approaches include the bubble population-balance model, network/percolation theory, catastrophe theory, and filtration theory. All were developed for modeling polyhedral foam except for the filtration theory, which was developed for ball foam (microfoam).

Although disagreement exists as to the best approach for modeling foam displacement, there is general acceptance of principles used to guide model development (Zhou and Rossen 1995). For example, it is generally accepted that foam is not treated as a separate phase in porous media. Additionally, agreement exists on the concept that water mobility is the same function of water saturation with foam as without foam (Kahtib et al. 1988) and that gas mobility in foam is controlled by foam texture (Falls et al. 1989).

Foam simulators do not need to be mechanistic to be capable of accurately describing foam transport. However, Kovscek (1998) suggested that successful models of foam transport at the field scale should have the following attributes: 1) reduced gas mobility in the presence of foam; 2) non-Newtonian foam flow behavior; 3) foam property variability with surfactant concentration; and 4) an accurate representation of surfactant transport, partitioning, and adsorption. In summary, models need to predict foam transport while capturing the spatial and temporal variability of foam properties.

In Section 5.1 the major methods are reviewed in the order of empirical, semi-empirical, and mechanic models for modeling foam flow and displacement in porous media.

\subsection{Empirical Methods}

Foam mobility in porous media can be expressed in terms of relative permeability and effective viscosity in the same way that flow in porous media for Newtonian and non-Newtonian fluids is expressed (Tang and Kovscek 2006):

$$
\lambda_{f}=\frac{k k_{r f}}{\mu_{f}}
$$

For empirical methods of foam transport, either gas mobility or viscosity is altered in the presence of foam based on experimental results, field observations, and/or hypothesis or conjecture. Empirical expressions for gas mobility are usually expressed as a function of flow rates and/or surfactant concentration but make no explicit reference to foam texture.

\subsubsection{Mobility Reduction Factor Approach}

Using a relative permeability approach, the simplest technique for including the effects of foam in a simulator is through the use of a constant mobility reduction factor (MRF), which is commonly used in 
local steady-state modeling to describe foam rheology. Conceptually, the mobility reduction factor is defined for the same water saturation as:

$$
\begin{gathered}
M R F=\frac{\Delta P_{\text {with foam }}}{\Delta P_{\text {without foam }}}(\text { Rosman and Kam 2009) or } \\
M R F=\frac{\Delta P_{\text {with foam }}-\Delta P_{\text {without foam }}}{\Delta P_{\text {without foam }}} \text { (Hirasaki et al. 1997a, b) }
\end{gathered}
$$

where $\Delta \mathrm{P}$ is the pressure drop across the porous media. If the MRF is unity, or zero depending on the definition, then the injected foam is completely destroyed and the flow is equivalent to a typical gasliquid two-phase flow. Higher values of the MRF indicate the foam is more finely textured and stronger.

According to Kovscek (1998), an MRF has been incorporated in UTCHEM (Hirasaki et al. 1997a, b), a chemical flood simulator developed by the University of Texas at Austin. In this simulator, the gas permeability is divided by a constant value:

$$
k_{r f}\left(S_{g}\right)=\frac{k_{r g}\left(S_{g}\right)}{1+M R F}
$$

If the foam is very strong, the MRF is very large. For weak foams, the MRF is small. In this model, a threshold surfactant concentration must be exceeded before the gas mobility is modified. The weakness in employing a constant MRF is that the permeability reduction is a dynamic process, and it varies with gas velocity and surfactant concentration.

Another way to use the MRF approach is to tabulate MRF as a function of surfactant concentration, water saturation/pressure, and gas velocity. According to Kovscek (1998), this approach was applied to the ECLIPSE 200 (Geoquest) simulator. In the STARS simulator (Kular et al.1989; Mohammadi and Coombe 1992; Mohammadi et al. 1993), the MRF of foam was dependent on surfactant concentration, oil saturation, and gas velocity and was represented by

$$
M R F=M\left(\frac{C_{s}}{C_{s}^{\max }}\right)^{e_{s}}\left(\frac{S_{o}^{\max }-S_{o}}{S_{o}^{\max }}\right)^{e_{o}}\left(\frac{N_{c}}{N_{c}^{\max }}\right)^{e_{v}}
$$

(Kovscek 1998) where $\mathrm{C}_{\mathrm{s}}$ is the aqueous concentration of surfactant, $\mathrm{S}_{\mathrm{o}}$ is the oil saturation, $e$ are exponents, superscript max refer to the reference or maximum values of variables, and $\mathrm{N}_{\mathrm{c}}$ is a capillary number

$$
N_{c}=\frac{k \nabla p}{\sigma}
$$

where $\sigma$ is the gas/liquid interfacial tension. Equation [5.4] does not account for the impacts of liquid velocity and capillary pressure on MRF. 
The empirical method is also used in the UTCOMP simulator, which was developed at the University of Texas at Austin (Rossen et al. 1994; Shi and Rossen 1996; Kovscek 1998). If surfactant is present and capillary pressure is low, gas mobility is small via a reduced gas relative permeability. There is no velocity dependence on reduced mobility.

\subsubsection{Effective Viscosity Approach}

Another empirical approach to simulate foam transport is to modify the gas-phase relative permeability by using an effective viscosity of the gas phase and thereby reducing gas-phase mobility when foam is present. Marfoe et al. (1987) employed this approach with a function of surfactant concentration, $\mathrm{C}_{\mathrm{s}}$, aqueous-phase saturation, $S_{w}$, and gas-phase velocity, $\mathrm{v}_{\mathrm{g}}$ :

$$
\mu_{f}=\mu_{g}\left[1+0.01 C_{s}\left(S_{w}-S_{w r}\right) f\left(v_{g}\right)\right]
$$

where $S_{w r}$ is the residual aqueous saturation. The constant, 0.01, used in Eq. [5.6] gives a five- to ten-fold increase in gas viscosity. A larger value may be used if greater reduced mobility is expected. For their study, Marfoe et al. (1987) set $\mathrm{f}\left(\mathrm{v}_{\mathrm{g}}\right)=1$.

Islam and Ali (1990) implemented a more complex effective viscosity function including permeability and oil saturation expressed as:

$$
\mu_{f}=\mu_{g} \frac{1+D f_{c}\left(S_{w}-S_{w r}\right) f_{k}+f_{p}}{1+E S_{o}^{2}}
$$

where $f_{c}, f_{k}$ and $f_{p}$ are functions of surfactant concentration, permeability, and pressure gradient, respectively, and $D$ and $E$ are constants. The $f_{c}, f_{k}$, and $f_{p}$ functions were determined by history matching and experimental observations.

\subsection{The Semi-Empirical Fractional Flow Theory}

In the semi-empirical fractional flow theory, the physics of foam displacement are described mechanistically, but simplifying assumptions are used to develop fractional flow curves that account for impacts due to the presence of foam.

The fractional flow theory describes the physics of miscible and immiscible displacements in porous media in which the governing partial differential equations are solved analytically by a mathematical technique called the method of characteristics. The fractional flow curves, constructed theoretically by plotting water fractional flow $\left(f_{w}\right)$ on the $y$-axis and water saturation on the $x$-axis, were first presented and solved by Buckley and Leverett (1942). Since then, a number of studies have further developed the theory to account for a wide range of applications in oil recovery (Dake 1978; Pope 1980). Fractional flow solutions for foam applications have been presented by Rossen and Zhou (1995) and Zhou and Rossen (1995).

The approach is considered semi-empirical due to the required simplifying assumptions including onedimensional displacement, Newtonian viscosity, no viscous fingering, no capillary-pressure gradients, 
negligible gravitational impact, no physical dispersion, incompressible phases, and immediate attainment of local steady state. Despite these simplifications, many of the assumptions can be relaxed to some extent, and the solutions can still capture complex displacement mechanisms (Dholkawala et al. 2007).

In the following, for the convenience of readers and the continuity of the theory, the original fractional flow model for the oil-water system (Buckley and Leverett 1942) is introduced before the fraction-flow models for systems containing foam are presented.

\subsubsection{The Fractional Flow Model for a Oil-Water System}

The mathematical basis for the calculation of the simultaneous flow of the oil-water flow is completed by the introduction of the relevant conservation laws. A conservation law for each fluid, together with the flux equations, results in two strongly coupled partial differential equations. Considering the relation $P_{c}\left(S_{w}\right), k_{r w}\left(S_{w}\right), k_{r n w}\left(S_{w}\right)$, and the identity $S_{w}+S_{n w}=1$, these equations must be solved simultaneously subject to the appropriate boundary and initial conditions. For a horizontal unsteady flow of incompressible fluids, the gravity effect can be ignored and the general differential equation for water flow is (Fokas and Yortsos 1982; McWhorter and Sunada 1990; McWhorter and Kueper 1996):

$$
\phi \frac{\partial S_{w}}{\partial t}=\frac{\partial}{\partial}\left(D \frac{\partial S_{w}}{\partial x}\right)-q_{t} d \frac{f_{w}}{d S_{w}} \frac{\partial S_{w}}{\partial x}
$$

where

$$
\begin{aligned}
& f_{w}\left(S_{w}\right)=\left(1+\frac{k_{r n w} \mu_{w}}{k_{r w} \mu_{n w}}\right)^{-1} \\
& D\left(S_{w}\right)=-\frac{k k_{r n w} f_{w}}{\mu_{n w}} \frac{d P_{c}}{d S_{w}} \\
& q_{t}(t)=q_{w}(x, t)+q_{n w}(x, t)
\end{aligned}
$$

where $f_{w}$ is the fractional flow of water, and $q_{t}$ is the total flux of the aqueous and nonaqueous phases. The classical solution for the unsteady horizontal flow presented by Buckley and Leverett (1942) recognized that when the injection rate is large, the second term on the right-hand side of Eq. [5.8] is dominant, and the first term can be neglected. This indicates that the flow due to capillary gradient is negligible. Replacing $\partial S_{w} / \partial t$ by $\left(\partial S_{w} / \partial x\right)(d x / d t)$ cancels the term $\partial S_{w} / \partial x$ to produce a first-order differential equation:

$$
\phi \frac{d x}{d t}=-q_{t} f_{w}^{\prime}
$$

where

$$
f_{w}^{\prime}=d f_{w} / d S_{w}
$$


Hence,

$$
x\left(S_{w}, t\right)=\frac{f_{w}^{\prime}}{\phi} \int_{0}^{t} q_{t}(\tau) d \tau
$$

For an injection with a constant rate q, Eq. [5.14] simplifies as

$$
x\left(S_{w}, t\right)=\frac{f_{w}^{\prime} q t}{\phi}
$$

For the given $q$, $\phi$ and $f_{w}$, an $x$ value can be obtained for a certain time $t$. The function $f_{w}\left(S_{w}\right)$ is typically S-shaped, so for a given $f_{w}, S_{w}$ is not single valued. This physically untenable result is circumvented by the Welge (1952) tangent construction in which $f_{w}$ is replaced by the two-part function:

$$
\begin{gathered}
f_{w}\left(S_{w}\right)=\left(1+\frac{k_{r n w} \mu_{w}}{k_{r w} \mu_{n w}}\right)^{-1} \text { if } \mathrm{S}_{\mathrm{w}} \geq \mathrm{S}_{\mathrm{b}} \\
f_{w}\left(S_{w}\right)=f\left(S_{b}\right)\left(\frac{S_{w}-S_{i}}{S_{b}-S_{i}}\right) \text { if } \mathrm{S}_{\mathrm{i}} \leq \mathrm{S}_{\mathrm{w}}<\mathrm{S}_{\mathrm{b}}
\end{gathered}
$$

where $S_{i}$ is the initial saturation and $S_{b}$ is the saturation corresponding to the point the tangent line is drawn. The use of the Welge (1952) tangent construction produces a shock front.

A major assumption with the fractional flow model is the neglect of the capillary gradient. This is probably acceptable when the aqueous saturation is high and the capillary gradient is small. However, at low aqueous saturations (e.g., slightly greater than the critical saturation $\mathrm{S}_{\mathrm{w}}{ }^{*}$ ), the capillary gradient can be very significant.

\subsubsection{The Fractional Flow Model for a Foam-Water System}

The fractional flow solution for foam-water systems was presented by Rossen and Zhou (1995). In addition to the assumptions described above, they further assumed that a mobility reduction factor can be defined to represent foam mechanisms, and the foam-containing gas is incompressible. Despite these assumptions, Rossen et al. (1999) shows that the fractional flow theory is still able to explain complex mechanisms involved in foam displacements in porous media. Dholkawala et al. (2007) summarized the fractional flow model as given below.

In the foam-aqueous phase system, the foam was treated as the non-aqueous phase and the fractional flow of phase $\gamma$ can be described by

$$
f_{\gamma}=\frac{q_{\gamma}}{q_{t}}
$$


In Zhou and Rossen (1995), foam generation and coalescence were not considered. The model was later modified by Dholkawala et al. (2007) to include foam generation and coalescence of dynamic processes. Details of these two models are given below.

\subsubsection{The Zhou and Rossen (1995) Fixed- $P_{c}{ }^{*}$ Model}

According to Zhou and Rossen (1995), in some cases, the critical or limiting capillary pressure $P_{c}{ }^{*}$ is independent of gas and liquid flow rates; then the aqueous critical saturation $S_{w}{ }^{*}$ and mobility $\lambda_{w}{ }^{*}$ are likewise constant and independent of pressure gradient and gas and liquid flow rates. Zhou and Rossen (1995) called this the fixed- $\mathrm{P}_{\mathrm{c}}{ }^{*}$ case. They further assumed that at $S_{w}{ }^{*}$ foam quality, $f$, is equal to the fractional flow of gas. Hence, for these foams, gas mobility is (Zhou and Rossen 1995)

$$
\lambda_{g}=\lambda_{w}^{*} \frac{q_{g}}{q_{w}}=\lambda_{w}^{*}\left(\frac{1-f_{w}}{f_{w}}\right)=\lambda_{w}^{*}\left(\frac{f}{1-f}\right) \text { at } \mathrm{S}_{\mathrm{w}}=\mathrm{S}_{\mathrm{w}}{ }^{*}
$$

Equation [5.18] indicates that gas mobility at $P_{c}{ }^{*}$ is a function of $f_{w}$ (i.e., a function of foam quality) and $S_{w}{ }^{*}$ only.

Some data imply that $P_{c}{ }^{*}, S_{w}{ }^{*}$, and $\lambda_{w}{ }^{*}$ depend on flow rates of gas or liquid (Khatib et al. 1988; Falls et al. 1989; Friedman et al. 1991; Fisher et al. 1990). The fixed- $P_{c}{ }^{*}$ model is only approximately correct for these foams. Moreover, the fixed $P_{c}{ }^{*}$ model describes foam at steady state and may apply to the period of foam generation or at the leading edge of an advancing foam front (Zhou and Rossen 1995).

\subsubsection{The Dholkawala et al. (2007) Model}

A modified version of the mechanistic description of in-situ lamella creation is used, which accounts for the pressure gradient $(\nabla P)$ and water saturation $\left(S_{w}\right)$ as

$$
r_{g}=c_{g} S_{w}(\nabla P)^{a}
$$

where $r_{g}$ is the rate of lamella creation, $C_{g}$ and $a$ are the model parameters, and $\nabla P$ is the pressure gradient. Foam coalescence is governed by $\mathrm{P}_{\mathrm{c}}{ }^{*}$ or $\mathrm{S}_{\mathrm{w}}{ }^{*}$ :

$$
r_{c}=c_{c} n_{f}\left(\frac{1}{S_{w}+S_{w}^{*}}\right)^{b}
$$


where $r_{c}$ is the rate of foam coalescence, $n_{f}$ is the number of lamellae per unit volume of foam, $C_{c}$ and $b$ are model parameters. The interplay between lamella creation and coalescence determines the number of lamellae and hence governs the foam texture. By equating Eqs. [5.19] and [5.20], foam texture $\left(\mathrm{n}_{\mathrm{f}}\right)$ at local steady state becomes

$$
n_{f}=\left(\frac{c_{g}}{c_{c}}\right)\left(S_{w}-S_{w}^{*}\right)^{b} S_{w}(\nabla P)^{a}
$$

The shear-thinning effective viscosity of foam suggested by Hirasaki and Lawson (1985) is given as:

$$
\mu_{f}=\mu_{g}+\frac{C_{f} n_{f}}{q_{f}^{1 / 3}}
$$

where $\mu_{g}$ is the gas viscosity in the absence of foam, and $C_{f}$ is a model parameter accounting for gas viscosity in the presence of foam. Darcy's law is used to describe the flow of foam:

$$
q_{f}=\frac{k k_{r f}^{0} \nabla P}{\mu_{f}}
$$

where $k_{r f}^{0}$ is the gas relative permeability in the absence of foam. In local steady-state modeling, one can determine $\mu_{f}$ and $q_{f}$ by simultaneously solving Eqs. [5.22] and [5.23] and constructing fractional flow curves using [5.17]. Once a fractional flow curve is constructed, a solution can be attained for the initial and injection conditions. This foam model has been shown to successfully fit the three foam states (weak, intermediate, and strong) and the two steady-state strong-foam regimes (low-quality and high-quality) observed in laboratory experiments (Alvarez et al. 2001, Gauglitz et al. 2002).

Dholkawala et al. (2007) showed that when the fractional flow curves are constructed using mechanistic descriptions of foam rheology in porous media, the solutions quantitatively show how the shape of the fractional flow curve changes with injection velocity. The fractional flow theory could explain the weakfoam state at low injection velocity, the strong-foam state at high injection velocity, the transition from the weak-foam to the strong-foam state, the hysteresis associated with foam generation, and the effect of foam quality on foam generation. Dholkawala et al. (2007) demonstrated that foam rheology, similar to catastrophe theory (see Section 5.3.2), is a multi-valued problem when the liquid and gas flow rates are fixed, but a single-valued problem when the pressure gradient is fixed.

\subsubsection{The Fractional Flow Model for the Gas-Oil-Water System}

Rosman and Kam (2009) recently developed a model that describes foam rheology in the presence of oil using three-phase, three-component fractional flow theory. Schramm (1994) noted that an increase in oil saturation has a destabilizing effect and reduces foam strength by lowering the MRF. This occurs because there is a threshold oil saturation above which foams are not stable. Different surfactant formulations also react differently to different oil types. 
Although oil is known to have detrimental effects on foam in porous media, few studies have included its impact on foam floods (e.g., Fisher et al. 1990, Law et al. 1992). The generalized approach for accounting for the effect of oil on foam introduces an empirical constant when the oil saturation is greater than zero. Rosman and Kam (2009) present a similar approach where three-phase fractional flow curves are constructed based on the same mathematical formulations developed for the immiscible two-phase process. Assuming gravity and capillary effects are negligible, the generalized fractional flow equation for a three-component, three-phase (gas-oil-water) flow in a horizontal system is given as

$$
f_{\gamma}=\frac{k_{r \gamma} / \mu_{\gamma}}{k_{r w} / \mu_{w}+k_{r o} / \mu_{o}+k_{r g} /\left(\mu_{g} M R F\right)}
$$

where subscripts $w, o$ and $g$ represent water, oil, and gas. An $M R F$ is necessary in this formulation to account for the reduction in gas mobility in the presence of foam. The fraction flow of phase $\gamma$ is then a unique function of phase saturations.

The volume balance for fractional flow (or mass balance for incompressible phases) is given as $f_{w}+f_{o}+f_{g}=1$ with saturations summing to one as well, $S_{w}+S_{o}+S_{g}=1$.

Using mass conservation equations to describe foam transport, the solution for spreading waves is

$$
\left(\frac{d x_{D}}{d t_{D}}\right)_{s_{\gamma}}=\left(\frac{d f_{\gamma}}{d S_{\gamma}}\right)_{s_{\gamma}}=v_{s_{\gamma}}
$$

where $v_{s_{\gamma}}$ is dimensionless velocity, and $x_{D}$ and $t_{D}$ are dimensionless distance and dimensionless time, respectively. For shock waves, Eq. [5.25] is expressed as

$$
v_{s_{\gamma}}=\frac{\Delta f_{\gamma}}{\Delta S_{\gamma}}
$$

where $\Delta f_{\gamma}$ and $\Delta S_{\gamma}$ are discontinuous changes in fractional flow and saturation of phase $\gamma$ across the shock. A key concept in describing multi-component, multiphase displacement in a porous medium is "coherence," which requires that all dependent variable at any point in space and time have the same velocity. Hence,

$$
\frac{d f_{w}}{d S_{w}}=\frac{d f_{o}}{d S_{o}}=\frac{d f_{g}}{d S_{g}}=\sigma
$$


where $\sigma$ is the characteristic direction. The method of characteristics can be used to convert the derivatives of Eq. [5.25] into an eigenvalue problem that describes fast and slow moving saturation waves. Monotonically decreasing velocity from injection to initial condition is a constraint of fractional flow theory. Hence, the high-velocity path is first, followed by the low-velocity path. Relative permeability functions for this model were assumed to be

$$
\begin{gathered}
k_{r w}=k_{r w}^{0}\left(\frac{S_{w}-S_{w r}}{1-S_{w r}-S_{o r w}}\right)^{n_{w}} \\
k_{r g}=k_{r g}^{0}\left(\frac{1-S_{w}-S_{o}-S_{g r}}{1-S_{w r}-S_{g r}}\right)^{n_{g}} \\
k_{r o}=k_{r o}^{0}\left\{\left(\frac{k_{r o w}}{k_{r o}^{0}}+k_{r w}\right)\left(\frac{k_{r o g}}{k_{r o}^{0}}+k_{r g}\right)-\left(k_{r w}+k_{r g}\right)\right\}
\end{gathered}
$$

where

$$
\begin{gathered}
k_{\text {row }}=k_{r o}^{0}\left(\frac{1-S_{w}-S_{o r w}}{1-S_{\text {orw }}-S_{w r}}\right)^{n_{o w}} \\
k_{\text {rog }}=k_{r o}^{0}\left(\frac{S_{o}+S_{w}-\left(S_{o r g}+S_{w r}\right.}{1-\left(S_{\text {org }}+S_{w r}\right)-S_{g r}}\right)^{n_{o g}}
\end{gathered}
$$

$k_{r w}^{0}, k_{r o}^{0}$ and $k_{r g}^{0}$ are the end-point relative permeability values of water, oil and gas, $S_{o r w}$ and $S_{o r g}$ are residual oil saturation in a water-oil and gas-oil system, and $n_{w}, n_{o w}$ and $n_{o g}$ are the relative permeability exponents.

Rosman and Kam (2009) applied this model to a two-layer system to determine the effectiveness of foam diversion (i.e., preferentially enter low-permeability layers) for a system with mixed permeabilities. The results demonstrated that the frontal velocities and saturation profiles need to be considered to evaluate foam diversion. To this end, the MRF must be large in both layers to form piston-like displacements. Moreover, the MRF in the low-permeability layer must be small relative to the MRF in the highpermeability layer for the displacement front in the low-permeability layer to catch up with the front in the high-permeability layer.

\subsubsection{Fraction Flow Model Limitations}

Due to the assumptions described above, the fraction flow model may be unsuitable for modeling foam flooding because fractional flow theory is approximate when applied to compressible phases. Severe extrapolations from available data are needed to fit model parameters, and strong foam behavior is not, in general, a unique function of fractional flow. Constructing fractional flow curves for foam flow in porous media may be inappropriate because absolute flow rates determine foam-flow behavior. 


\subsection{Mechanistic Methods}

A major challenge to developing a mechanistic foam simulator is tracking changes in foam texture. These changes result from dynamic mechanisms of in-situ lamella creation and coalescence, which in turn, results in changes in the gas mobility and pressure gradient. The concept of tracking the bubble population has been used in many methods for foam simulation.

\subsubsection{Bubble Population-Balance Model}

Reduced gas mobility in the presence of foam is due to the following mechanisms: 1) stationary or trapped foam that blocks channels that would otherwise carry gas, 2) flowing bubble trains that encounter drag from pore walls and constrictions (Falls et al. 1989), and 3) viscous and capillary forces that constantly rearrange the gas-liquid interfacial area of a flowing foam bubble (Hirasaki and Lawson 1985). Gas mobility in the presence of foam critically depends on the foam-bubble size, which may vary with permeability, porosity, surfactant type/concentration, and the velocity of the liquid and gas.

Bubbles are molded and shaped by porous media because porosity reflects the relative abundance of foam germination and termination sites per unit volume of porous media (Chambers and Radke 1991; Ettinger and Radke 1992). Bubbles and lamellae are usually transported some distance and may be destroyed and then regenerated. When the local pressure gradient is insufficient to keep bubbles mobilized, other bubble trains may begin to flow. No single bubble or bubble train is conserved over the length of several pore bodies. Hence, foam texture arises from a balance between varied and complicated foam generation and destruction mechanisms, and bubble trains only exist in a time-averaged sense.

Because foam texture determines the resistance of flow in porous media, the direct incorporation of the role of foam texture into a simulator may be necessary for successful predictions of foam rheology. The population balance method quantifies the evolution of foam texture and gas mobility according to the bubble concentration. It is a mechanistic approach inasmuch as pore-level events are depicted in foam generation, coalescence, and constitutive equations. Key to the successful application of this approach is obtaining realistic rate expressions for the generation and coalescence of foam. By analogy to mass balance equations for solutes in a subsurface flow and transport simulator, a separate conservation equation is written for the concentration of foam bubbles.

\subsubsection{Bubble Generation and Coalescence}

A variety of algorithms have been used for describing lamella creation (bubble generation). Falls et al. (1988) described bubble generation as a function of capillary pressure, whereas bubble generation was triggered once a minimum flow rate was exceeded in the population balance model of Friedmann et al. (1991). Kam and Rossen (2003) described bubble generation as a simple function of the pressure gradient given as

$$
r_{g}=C_{g}(\nabla p)^{m}
$$

where $r_{g}$ is the rate of bubble generation, $\nabla p$ is the time-averaged pressure gradient, and $C_{g}$ and $m$ are model parameters. Others have described bubble generation based on snap-off at germination sites 
(Kovscek and Radke 1994; Bertin et al. 1998; Myers and Radke 2000), which are narrow pore throats located upstream of wide pore bodies. Kovscek (1993) extended the hydrodynamic analysis of Ransohoff et al. (1987) for constricted, cornered pores to include imposed wetting liquid flow and found that bubble generation by the snap-off mechanism could be expressed as being linearly proportional to liquid velocity and to gas velocity raised to a power less than unity:

$$
r_{g}=k_{1} v_{f}^{a} v_{w}^{b}
$$

where $k_{1}$ is the generation rate constant, $v_{w}=q_{w} \phi S_{w}$ is the local pore water velocity, $v_{f}=q_{f} \phi S_{f}$ is the local velocity of flowing foam, and $a$ and $b$ are power indices with the index $b$ close to unity. Equation [5.34] suggests that bubbles are produced only in the portion of the foam being transported.

The rate of lamella destruction (bubble coalescence) is still unknown, but it is generally agreed that lamella destruction should diverge toward infinity as the water saturation $\left(S_{w}\right)$ approaches the critical water saturation $\left(S_{w}{ }^{*}\right)$ or as the capillary pressure $\left(P_{c}\right)$ approaches the critical capillary pressure $\left(P_{c}{ }^{*}\right)$. At these critical values, foam is known to collapse or coalesce. Assuming divergence at $S_{w}{ }^{*}$, Kam and Rossen (2003) described the rate of bubble coalescence as

$$
r_{c}=C_{c} n_{f}\left(\frac{1}{S_{w}-S_{w}^{*}}\right)^{n}
$$

where $r_{c}$ is the rate of foam coalescence, $n_{f}$ is number of lamellae in a unit volume, and $C_{c}$ and $n$ are model parameters. Others have included a term for the rate of lamella transport into termination sites, where lamellae are destroyed. Ettinger and Radke (1992) adopted an expression where foam lamellae are destroyed in proportion to their flux (i.e., $v_{f} n_{f}$ ) into termination sites:

$$
r_{c}=k_{-1}\left(S_{w}\right) v_{f} n_{f}
$$

where $k_{-1}$ is the coalescence rate constant that varies strongly with local aqueous-phase saturation and surfactant formulation/concentration. This is expressed as

$$
k_{-1}\left(S_{w}\right)=k_{-1}^{0} \frac{1-S_{w}}{S_{w}-S_{w}^{*}}
$$

where $k_{-1}{ }^{0}$ is a scaling constant. Consistent with divergence at $S_{w}{ }^{*}$, the coalescence rate increases monotonically as the porous medium desaturates. Because of the steepness of the coalescence rate with $S_{w}$, changes in gas or liquid velocity have little effect on the steady-state saturation (Khatib et al.1988). Ettinger and Radke (1992) used the same rate expression to describe the rate of bubble division, the second mechanism for creating foam. This rate is proportional to the flux of lamellae into the division sites. Both rate constants are small with high values of $S_{w}$ because fewer division sites are available. 
In Kovscek et al. (1995, 1997), the coalescence rate was written in terms of capillary pressure as

$$
k_{-1}\left(S_{w}\right)=k_{-1}^{0}\left(\frac{P_{c}}{P_{c}^{*}-P_{c}}\right)^{2}
$$

The critical pressure head $P_{c}{ }^{*}$ is a function of surfactant concentration and may be estimated in different formulations:

$$
\begin{gathered}
P_{c}^{*}=P_{c}^{*}, \max \tanh \left(\frac{C_{s}}{C_{s}^{0}}\right)(\text { Kovscek et al. 1995) } \\
P_{c}^{*}=P_{c}^{*} \text { max }\left(\frac{C_{s}}{C_{s}^{0}}\right) \text { (Kovscek et al. 1997) }
\end{gathered}
$$

where $P_{c}{ }^{*}$,max is a limiting value of $P_{c}{ }^{*}$ at a reference surfactant concentration, $C_{s}{ }^{0}$, and $C_{s}$ is surfactant concentration. This function allows $P_{c}{ }^{*}$ to increase rapidly and smoothly from zero when $\mathrm{C}_{\mathrm{s}}=0$ to the maximum $P_{c}{ }^{*}$ max when $C_{s} \geq C_{c}{ }^{0}$.

In practice, it is difficult to distinguish between bubble generation and coalescence when writing mechanistic rate expressions. Hence, the two processes are often combined and expressed as one equation. Because gas diffusion coarsening of the trapped phase is not currently well understood, it is not often included in rate equations.

\subsubsection{Trapped Foam}

Trapped or stationary gas can contribute significantly to the reduction of foam mobility. Gas tracer experiments with steady-state foam flow measure the fraction of trapped gas to vary between $85 \%$ and 99\% (Gillis and Radke 1990; Friedmann et al. 1991). Coarsening of trapped foam, the process of larger bubbles growing at the expense of smaller ones, can occur in porous media via gas diffusion when lamellae reach the same pore-throat (Kovscek and Radke 1994). In general, the fraction of trapped foam to flowing foam, $\chi_{t}=S_{t} / S_{f}$, is a function of pressure gradient, capillary pressure, and pore geometry. Kovscek and Radke (1994) described the trapped fraction of foam as a function of the trapped texture, $n_{t}$ :

$$
\chi_{t}=\chi_{t}^{\max } \frac{\beta n_{t}}{1+\beta n_{t}}
$$

where $\chi_{t}^{\text {max }}$ is the maximum fraction of trapped foam and $\beta$ is a trapping parameter. 


\subsubsection{Conservation and Rate Equations}

The rate equations for bubble generation $\left(r_{g}\right)$ and coalescence $\left(r_{c}\right)$ can be incorporated into subsurface simulators using mass balance equations for the gas and aqueous phases. For example, a mass balance equation for a one-dimensional medium can be given as (Kovscek and Radke 1994):

$$
\frac{\partial\left(\phi \rho_{\gamma} S_{\gamma}\right)}{\partial t}=-\frac{\partial\left(\rho_{\gamma} q_{\gamma}\right)}{\partial x}+Q_{\gamma}, \gamma=g \text { or } l
$$

where $q_{\gamma}$ is the Darcy velocity and $Q_{\gamma}$ is the source/sink term for gas or water. In Eq. [5.41], it is assumed that there is no exchange of water mass between the two phases. The mass balance equation for a surfactant can be expressed as:

$$
\frac{\partial\left[\phi\left(C_{s} S_{w}+\Gamma_{s}\right)\right.}{\partial t}=-\frac{\partial\left(q_{w} C_{s}\right)}{\partial x}+Q_{s}
$$

where $C_{s}$ is the concentration of surfactant in the aqueous phase, $\Gamma_{s}$ is the amount of surfactant adsorption on porous media surfaces, and $Q_{s}$ represents the surfactant source term. Note that the mass of surfactant in the gas phase is not considered because it is generally considered negligible compared to the surfactant mass in the aqueous phase. Because the mobility of the foam phase is a strong function of texture, a conservation statement that accounts for the evolution of foam bubble size is needed (Patzek 1988). The transient population-balance on the mean bubble size can be written as (Patzek 1988; Falls et al. 1988; Kovscek and Radke 1994)

$$
\frac{\partial\left[\phi\left(S_{f} n_{f}+S_{t} n_{t}\right)\right.}{\partial t}=-\frac{\partial\left(q_{f} n_{f}\right)}{\partial x}+\phi S_{g}\left(r_{g}-r_{c}\right)+Q_{b}
$$

where $Q_{b}$ is a source/sink term for foam bubbles, and subscripts $f$ and $t$ represent flowing and trapped foam. The parameter $n_{t}$ represents the number of trapped or stationary foam bubbles. The first term of the time derivative is the rate at which flowing foam texture becomes finer or coarser per unit volume of porous media, and the second term is the net rate at which foam bubbles trap. The spatial term on the right-hand side of the equation tracks the convection of flowing foam bubbles. At steady state, the rate of bubble generation balances the rate of bubble coalescence $\left(r_{g}=r_{c}\right)$. Kinetic expressions, such as those given in Section 5.3.1.1, can be used for $r_{g}$ and $r_{c}$.

\subsubsection{Limitations}

Although the method is mechanistic and portrays well-documented pore-level events, the expressions are generalized to represent bulk behavior and contain parameters that may be difficult to obtain, especially at the field-scale. Moreover, the population balance model neglects the surfactant mass in the gas phase, which may be important for unsaturated subsurface applications. 


\subsubsection{Catastrophe Theory}

Catastrophe theory is a branch of bifurcation theory that studies and classifies phenomena characterized by sudden shifts in behavior arising from small changes in circumstances. Small changes in certain parameters of a nonlinear system can cause equilibria to appear or disappear, leading to large and sudden changes of system behavior.

Kam et al. (2007) discussed the foam catastrophe problem in detail, saying that $S_{w}$ in the high-quality regime is extraordinarily close to $S_{w}{ }^{*}$ and it is extremely difficult to avoid reaching the singularity in $r_{c}$ (the rate of lamella coalescence) at $S_{w}{ }^{*}$ since the simulation would require extraordinarily short time steps to avoid the singularity. The fractional flow analysis cannot adequately describe the dynamics of foam mechanism, especially near the leading edge of from front, due to its local-steady-state assumption. It is questionable whether simulators based on the population balance model can successfully integrate the catastrophe theory and the two strong-foam regimes.

Kam (2008) for the first time resolved the issues on the instability and divergence of numerical simulation that take place near the physical discontinuity in foam simulation and presented how to build a mechanistic foam simulator consistent with foam catastrophe theory and two steady-state strong-foam regimes.

In Kam (2008), the bubble population in the gas phase in 1-D space is expressed as

$$
\frac{\partial\left[\phi\left(S_{f} n_{f}\right)\right.}{\partial t}=-\frac{\partial\left(q_{f} n_{f}\right)}{\partial x}+\phi S_{g} r
$$

where $r$ is the net change of $n_{f}$ per unit time:

$$
r=\left\{\begin{array}{cc}
r_{c}-r_{g} & \text { if } S_{w}>S_{w}^{*} \\
0 & \text { if } S_{w}<S_{w}^{*}
\end{array}\right.
$$

The rate of lamella breakage $\left(r_{c}\right)$ is described by $S_{w}$ and flows up at $S_{w}{ }^{*}$ (Aronson et al.1994; Khatib et al. 1988; Kam 2008)

$$
r_{c}=C_{c} n_{f}\left(\frac{S_{w}}{S_{w}-S_{w}^{*}}\right)^{n} \text { if } S_{w}>S_{w}^{*}
$$

The lamella creation rate, $r_{g}$, is generally considered as $r_{g} \propto \nabla P^{m}, m>1$. Kam (2008) showed that it is necessary to select an appropriate lamella-creation function to ensure the stability and convergence of the numerical solution. Specifically, 1) the lamella-creation function should increase rapidly above a certain minimum pressure gradient for lamella mobilization and division to kick off active bubble generation, and 2) the function should also reach a plateau at high $\nabla \mathrm{P}$ at which foam rheology is governed by bubble 
coalescence rather than bubble generation. Previous lamella-creation functions have only satisfied the first constraint, causing a singularity to occur when $S_{w}$ approaches $S_{w}{ }^{*}$. To this end, Kam (2008) introduced the following function:

$$
r_{g}=\frac{c_{g}}{2}\left[\operatorname{erf}\left(\frac{\nabla P-\nabla P_{0}}{\sqrt{2}}\right)-\operatorname{erf}\left(\frac{-\nabla P_{0}}{\sqrt{2}}\right)\right]
$$

where $e r f$ is the error function. Foam texture at equilibrium can then be obtained in equating Eqs. [5.46] and [5.47]:

$$
n_{f}=\frac{c_{g}}{2 c_{c}}\left(\frac{S_{w}-S_{w}^{*}}{S_{w}}\right)^{n}\left[\operatorname{erf}\left(\frac{\nabla P-\nabla P_{0}}{\sqrt{2}}\right)-\operatorname{erf}\left(\frac{-\nabla P_{0}}{\sqrt{2}}\right)\right] \text { if } n_{f}<n_{f}^{\max }
$$

where $n_{f}^{\max }$ is the foam texture at minimum bubble size, which is determined from the average pore size.

Kam (2008) compared results obtained by the catastrophe theory to solutions obtained by the fractional flow theory (see Section 5.2). Although the results of the mechanistic simulator compare well to those obtained by the fractional flow theory, the actual displacement that resulted from a given injection condition could only be predicted by the dynamic simulations using the mechanistic simulator.

\subsubsection{Percolation Theory}

Typical reservoir simulations treat the flow as taking place in an averaged, Darcy-scale medium. However, simulations can also be carried out at the level of the network of pores and throats of the porous medium. Percolation theory allows this application by providing the ability to quantify connections of volumes, areas or line segments when arranged at random. When these line segments represent transport between neighboring pores, the statistics of their connectivity reveal information about the rate-limiting conductance of large systems (Hunt 2001). Percolation theory tracks connections, which makes it a logical choice for addressing spatial correlations. Thus, percolation theory has the strength of quantifying connections and emphasizing heterogeneity.

In percolation theory, a porous medium is represented as a network of pores (sites), which are connected by capillary tubes (bonds). Percolation theory deals with networks of sites and bonds where the topology of the system is determined by a number of connections that each site has with its neighboring sites. To illustrate how percolation theory might be applied to foam rheology, the model of Rossen and Gauglitz (1990) is described below.

\subsubsection{Cluster Size}

Rossen and Gauglitz (1990) approximated the interconnected pore network of a porous medium with a network of nodes and bonds. Nodes in the network represent pores, and the bonds connecting them represent pore throats. Missing nodes represent pores occupied by water; the removal of additional bonds represents blockage of pore throats by bubbles created by snap-off. Hence, the mobilization of lamellae from pore throats can refine foam texture. 
In the Rossen and Gauglitz (1990) model, conductivity across the network corresponds to gas permeability, and the potential drop across a missing bond represents the pressure drop across a blocked pore throat. They made several assumptions including no changes in gas and water saturations, equivalent conductivities among bonds, and random snap-off occurrence at pore throats. Since lamellae that block dead-end clusters are free to take the position in the throat where pressure gradient is at the maximum value, the pressure gradient across a lamellae is defined as

$$
\begin{aligned}
& \Delta P=G_{p} L \\
& G_{p}^{\min }=\Delta P^{\max } / L
\end{aligned}
$$

where $\Delta P^{\max }$ is the maximum pressure drop across the lamellae as it is displaced from the throat, and $G_{P}{ }^{\min }$ is the minimum pressure gradient required to displace it. No assumptions about the volumes of individual pores (nodes) are made. Although the authors refer to the fraction of pore throats occupied by gas as its saturation, it does not equal the volumetric saturation in the porous medium.

In the Rossen and Gauglitz (1990) model, a fraction of bonds, $f_{b}$, is randomly selected and assigned a conductivity of 1 , and the remaining bonds are assigned a conductivity 0 . As a function of $f_{b}$, analytical formulas are developed for the conductivity $\left[k_{r}\left(f_{b}\right)\right]$, the fraction of bonds conducting flow $\left[B_{c}\left(f_{b}\right)\right]$, and the total number of bonds $\left[B_{t}\left(f_{b}\right)\right]$ including the dead-end fractions of bonds. The percolation threshold, $f_{c}$, is the value of $f_{b}$ at which clusters of bonds link up to form an infinite network, and $k_{r}, B_{c}$ and $B_{t}$ become nonzero and is a function only of network topology. When gas invades a water-saturated porous medium, $f_{b}$ represents the fraction of pore throats large enough for gas to enter. $B_{t}\left(f_{b}\right)$ represents the fraction of throats occupied by gas, which establishes the conductivity, $k_{r}\left(f_{b}\right)$. The $f_{b}$ value at steady state before surfactant is introduced is denoted by $f_{b}^{0}$, and the actual gas saturation is denoted by $\mathrm{B}_{\mathrm{t}}\left(f_{b}{ }^{0}\right)$.

In a system with surfactant, when gas imbibition occurs, snap-off can reduce the value of $f_{b}$. The fraction of initially gas-occupied and accessible throats now blocked, $f_{\mathrm{sn}}$, is

$$
f_{s n}=\left(f_{b}^{0}-f_{b}\right) / f_{b}^{0}
$$

Though snap-off reduces the fraction of gas-occupied throats, it does not affect the occupation of pore bodies. Hence, the fraction of gas trapped, $B_{t r}$, is

$$
B_{t r}=\left[B_{t}\left(f_{b}^{0}\right)-B_{t}\left(f_{b}\right)\right] / B_{t}\left(f_{b}^{0}\right)
$$

Equation [5.51] applies only to the Bethe tree, an idealized network in which there is only one path between any two points (Larson and Davis 1982). If $B_{c}(f)$ is known, the average number of pores in a gas channel blocked by a single pore throat, $\bar{n}$, for any lattice can be determined by (Rossen 1988):

$$
\bar{n}=f_{b}^{0} \frac{d \ln B_{c}}{d \ln f_{b}}
$$


The percolation threshold is defined as the critical value of occupation probability. Near this value, the average number of pores in a gas channel blocked by a single pore throat is given as

$$
\lim _{f \rightarrow f_{b}^{c}} \bar{n}=f_{c} \gamma_{B}\left(f_{b}-f_{b}^{c}\right)^{-1}
$$

where $\gamma_{B}$ is the exponent for the scaling law for $B_{c}$ near $f_{c}$. For a Bethe tree of any coordination number, $\gamma_{B}$ $=2$ (Larson and Davis 1982), and for three-dimensional lattices, $\gamma_{B}=1.11$.

\subsubsection{Relating Cluster Size to $\Delta P$}

For any fraction of pore throats, $f_{b}$, there is a distribution of the number of connected pores, $n$, for the missing bonds that if opened, would contribute to flow. In general, the lamellae blocking the largest clusters have the largest $\Delta P$ and thus are mobilized first. An estimate for the average cluster length in the direction of flow, $L$, is

$$
L \cong \frac{3}{2} \bar{n} l
$$

where $L$ is the length of a single pore. Although the exact relation between $L$ and $\bar{n}$ on three-dimensional lattices is unknown, tortuosity can be considered by treating clusters as random walks of $3 \bar{n}$ steps.

At the onset of foam mobilization, Darcy's equation relates the pressure gradient to the gas velocity. Using Eqs. [5.49], [5.52] and [5.13], the minimum gas velocity for mobilization, $v_{g}{ }^{\text {min }}$ is defined as

$$
v_{g}^{\min }=\frac{k k_{r}\left(f_{b}\right)}{\phi \mu_{g}} G_{p}^{\min }
$$

where $k$ is absolute permeability, and $k_{r}\left(f_{b}\right)$ represents relative permeability to gas.

Using the above percolation theory in a Bethe tree network, Rossen and Gauglitz (1990) found that the cluster size diverges to infinity as the percolation threshold is approached, where foam generation becomes easier. Because the proximity to the percolation threshold is related to the injected liquid-gas volume ratio and fluid viscosities, the minimum pressure gradient from the injected liquid fraction can be predicted. This model explains experimental reports of a minimum pressure gradient or injection rate required to generate foam. However, the theory does not predict the mobilization pressure gradient once flow begins.

Chen et al. (2006) pointed out that in the percolation model of Rossen and Gauglitz (1990), the assumption of a uniform pressure gradient on the pore network near the percolation threshold is not a valid assumption. The average cluster of gas blocked by lamellae diverges to infinity at the percolation threshold, whereas the number density of these clusters on the pore network approaches zero. Chen and Yortsos (2006) advanced the Rossen and Gauglitz (1990) network model development by modeling the three processes of lamella creation (leave behind, lamella division, and snap-off). Only foam-generation mechanisms were considered, and they did not include relationships describing lamella destruction. The study demonstrated that strong foam generation by lamella mobilization and division and capillary 
fluctuations is possible without the necessity of repetitive snap-off. Minimum pressure gradient calculations using snap-off yielded qualitatively similar results of Rossen and Gauglitz (1990) without assuming a uniform pressure gradient in the pore network.

As referenced by Chen and Yortsos (2006), Tanzil et al. (2002) proposed that foam generation depends on the pressure drop across the displacement front rather than on the pressure gradient. They also proposed a critical value of the capillary number based on the pressure drop across the displacement front to denote the threshold of foam generation. However, verifying this hypothesis is difficult in fixed-injection-rate experiments because the pressure drop increases once foam is generated. The measured pressure drop is as much a result as it is a cause of foam generation.

\subsubsection{Limitations}

The percolation (or network) models that allow replication of pore-level mechanisms have the disadvantage of requiring large amounts of computational time and providing results on only small grids. It seems unlikely that network or percolation models can be useful in transient displacements that demand tracking of saturation, surfactant concentration, and foam quality at the field or even laboratory scale. Percolation theory is more appropriate for the study of foam at the pore scale.

\subsubsection{The Filtration Theory for Ball Foam}

Unlike other modeling methods, this modeling approach is specifically for ball foam, which is also termed microfoam or CGA. Ball foam is much denser than polyhedral foam, and hence, the former behaves more like a liquid while the latter is more like a gas. Due to its small bubble size, which is equivalent to that of colloids, Wan et al. (2001) treated CGA transport in porous media as a special case of colloidal transport based on the filtration theory:

$$
R \frac{\partial n_{f}}{\partial t}=D \frac{\partial^{2} n_{f}}{\partial x^{2}}+v_{w} \frac{\partial n_{f}}{\partial x}-k_{a} n_{f}
$$

where $n_{f}\left[\mathrm{~L}^{-3}\right]$ is bubble density (microbubble concentration), $R$ is the retardation factor for local sorption equilibrium, $D\left[\mathrm{~L}^{2} \mathrm{~T}^{-1}\right]$ is the dispersion coefficient ( $D=\alpha_{L} v_{w}$, where $\alpha_{\mathrm{L}}$ is the longitudinal dispersivity), and $k_{a}\left[\mathrm{~T}^{-1}\right]$ is the pseudo first-order rate coefficient for attachment.

The attachment coefficient can be related to the filter coefficient, $\lambda\left[\mathrm{L}^{-1}\right]$, used in the filtration theory (Yao et al. 1971):

$$
k_{a}=v_{w} \lambda=v_{w}\left\lfloor 1.5(1-\phi) \alpha \eta / d_{g}\right\rfloor
$$

where $d_{g}$ is the diameter of the filter media (collector), $\eta$ is the ratio of the rate at which colloids strike the single collector to the rate at which colloids flew toward the collector, and $\alpha$ is the fraction of collisions that results in attachment of the particle to the collector. Under favorable surface interactions between the particle and the collector that result in a net attractive force, $a$ approaches unity, while under unfavorable surface interactions resulting in a repulsive force barrier, $\alpha$ approaches zero. 
Rajagopalan and Tien (1976) developed the following approximate expression for $\eta$ as a function of several dimensionless numbers (Wan et al. 2001)

$$
\begin{aligned}
& \eta=\left[4 A_{s}^{1 / 3} N_{P e}^{-2 / 3}+A_{s} N_{L o}^{1 / 8} N_{R}^{15 / 8}+0.00338 A_{s} N_{G}^{1.2} N_{R}^{-0.4}\right] \\
& \text { where } \\
& N_{R}=d_{p} / d_{g} \\
& N_{P e}=U D_{c} /\left[k_{b} T\left(e \pi \mu d_{p}\right)\right] \\
& N_{G}=U_{p} / U \\
& N_{L o}=4 H /\left(9 \pi \mu d_{p}^{2} U\right) \\
& A_{s}=\frac{2\left(1-\gamma^{5}\right)}{2-3 \gamma+3 \gamma^{5}-2 \gamma^{6}} \\
& \gamma=(1-\phi)^{1 / 3}
\end{aligned}
$$

where $d_{p}[\mathrm{~L}]$ is particle diameter, $U\left[\mathrm{~L} \mathrm{~T}^{-1}\right]$ is the approach velocity, $k_{b}$ is the Boltzmann constant, $T[\mathrm{~K}]$ is temperature, $\mu\left[\mathrm{M} \mathrm{L}^{-1} \mathrm{~T}^{-1}\right]$ is fluid viscosity, $U_{p}$ is the particle settling velocity, and $H\left[\mathrm{M} \mathrm{L}^{-2} \mathrm{~T}^{-2}\right]$ is the Hamaker constant and usually assumed to be $10^{-20} \mathrm{~J}$.

For the buoyant-caused upward bubble movement, the buoyant rise velocity of bubbles is given by (Wan et al. 2001)

$$
U_{p}=g \rho_{f} d_{p}^{2} / 18 \mu_{f}
$$

where $\rho_{f}$ is fluid density. The collision of the efficiency factor, $\alpha$, can be estimated, after neglecting dispersion. according to the steady-state 1-D bubble concentration distribution over the length of the column ( $x=0$ to $L)$ (Wan et al. 2001)

$$
C / C_{0}=\exp (-\alpha L)
$$

where $C$ and $C_{0}$ are the effluent and influent concentrations.

Peters et al. (1994) very briefly described a foam simulator based on continuity principles, species conservation, and momentum balance for each phase. This model assumes a uniform foam bubble diameter, which is smaller than the equivalent diameter of the porous medium. The mean foam bubble size can be varied to assess its impact on foam flow in porous media. 



\subsection{Conclusions}

This section briefly summarizes the findings and challenges in using foam as an amendment-carrier for vadose zone remediation.

Often the properties of bulk foam are characterized and reported before foam is injected into a porous medium. These properties include critical micelle concentration, foam quality, stability, viscosity, surface tension, specific electric conductivity, foam disjoining pressure, etc. However, foam in porous media may have different properties than those of bulk foam.

Some work has been done for vadose zone remediation using either the micro-foam (colloidal gas aphrons) or the polyhedral foam in the laboratory scale. It appears that CGA suspensions provide better removal of the major chlorinated hydrocarbons present in oily waste, and foam can be used to enhance soil remediation under low injection pressures compared to other fluids such as liquid surfactant solutions. The concerns of vadose zone remediation using polyhedral foam include, but are not limited to, the lack of sufficient sustainability of foam and the possible excessive pressure gradient.

Major foam dynamic processes include foam destruction, formation, and entrapment. The transport of foam in porous media is complicated in that the number of lamellae present governs flow characteristics such viscosity, relative permeability, fluid distribution, and interactions between fluids. In a porous medium, there exists a critical capillary pressure above which lamellae cannot be sustained; a minimum pressure gradient is required to keep foam moving in a porous medium. The movement of foam can be described by the Darcy's law. However, the effective foam viscosity or mobility varies with foam texture and velocity. For the initially unsaturated sediment, there are limited experiment data and understanding of the impacts of flow dimensionality on foam dynamics and transport processes.

Three major approaches to modeling foam transport in porous media are the empirical, semi-empirical, and mechanistic. Mechanistic approaches can be complete in principle but it may be difficult to obtain reliable parameters, whereas empirical and semi-empirical approaches can be limited by the detail used to describe foam rheology and mobility. Critical assumptions have been imposed in the mechanistic and/or semi-empirical approaches. For example, it appears that the bubbles in the bubble-population model do not have mass. The fractional flow model neglects the impact of capillary gradient. The mass balance and capillary gradient can be critical for vadose zone remediation. For the polyhedral foam it is possible to find the relationships between foam mobility and foam quality/velocity through experiments, therefore the empirical approach may be appropriate to simulate foam transport in the initially unsaturated media. The transport of micro-foam in the initially saturated media has been modeled successfully with the filtration theory by treating the micro-bubbles as colloids. However, the stability of the micro-foam and its transport in the initially unsaturated media are not well-addressed in the literature. 



\subsection{References}

Aldana GAP. 2005. Effect of Surfactants on Drop Size Distribution in a Batch, Rotor-Stator Mixer. Ph.D. Dissertation, University of Maryland, College Park, Massachusetts.

Allred B and GO Brown. 1994. "Surfactant Induced Reductions in soil Hydraulic Conductivity.” Ground Water Monitoring Review, Spring, 174-184.

Aronson AS, V Bergeron, ME Fagan, CJ Radke. 1994. "The Influence of Disjoining Pressure on Foam Stability and Flow in Porous Media.” Colloids Surfaces A: Physicochem. Eng. Aspects 83:109-120.

Alvarez JM, H Rivas, and WR Rossen. 2001. "A Unified Model for Steady-State Foam Behavior at High and Low Foam Qualities.” SPE J. 325-333 (September).

Bernard GG, LW Holm, and WL Jacobs. 1965. "Effect of Foam on Trapped Gas Saturation and on Permeability of Porous Media to Water.” Soc. Pet. Eng. J. 5, 295-300.

Bertin HJ, MY Quintard and LM Castanier. 1998. "Development of a Bubble-Population Correlation for Foam-Flow Modeling in Porous Media.” SPEJ. 3(4):356-362.

Bikerman JJ. 1973. Foams. Springer-Verlag, Berlin.

Bretherton FP. 1961. “The Motion of Ling Bubbles in Tubes.” J. Fluid Mech. 10:166-188.

Buckley SE, and MC Leverett. 1942. "Mechanism of Fluid Displacement in Sands.” Trans. AIME 146, 107-116.

Chambers KT and CJ Radke. 1991. "Capillary Phenomena in Foam Flow Through Porous Media.” In NR Morrow (Ed.) Interfacial Phenomena in Petroleum Recovery. Marcel Dekker, New York. P. 191.

Chen M and YC Yortsos. 2006. "Pore-network study of the mechanisms of foam generation in porous media.” Physical Review E, 73, 036304.

Cheng L, SI Kam, M Delshad, and WR Rossen. 2002. "Simulation of Dynamic Foam-Acid Diversion Processes.” SPE J. 316-324 (September).

Chowdiah P, BR Misra, JJ Kilbane II, VJ Srivastava , and TD Hayes. 1998. "Foam Propagation Through Soils for Enhanced In-Situ Remediation.” Journal of Hazardous Materials 62:265-280.

Dake LP. 1978. Fundamentals of Reservoir Engineering. Elsevier, New York.

Derjaguin BV, and MM Kussakov. 1939. “Anomalous Properties of Thin Polymolecular Films.” V. Acta Physiocochim. URSS, 10(1):25-44.

Dholkawala ZF, HK Sarma, and SI Kam. 2007. "Application of Fractional Flow Theory to Foams in Porous Media.” Journal of Petroleum Science and Engineering 57, 152-165.

Dresel PE, N Qafoku, JP McKinley, JS Fruchter, CC Ainsworth, C Liu, ES Ilton, and JL Phillips. 2008. Geochemical Characterization of Chromate Contamination in the 100 Area Vadose Zone at the Hanford Site. PNNL-17674, Pacific Northwest National Laboratory, Richland, Washington. 
Ettinger RA. 1989. Foam Flow Resistance in Berea Sandstone. MS Thesis, University of California, Berkeley.

Ettinger RA and CJ Radke. 1992. "Influence of Foam Texture on Steady Foam Flow in Berea Sandstone.” Soc. Petr. Eng. Res. Eng. 7(1):83-90.

Falls AH, GJ Hirasaki, TW Patzek, PA Gauglitz, DD Miller, and T Ratulowski. 1988. "Development of a mechanistic foam simulator: The population balance and generation by snap-off.” Soc. Petr. Eng. Res. Eng. 3(3):884-892.

Falls AH, JJ Musters, and J Ratulowski. 1989. "The Apparent Viscosity of Foams in Homogeneous Beadpacks.” Soc. Petr. Eng. Res. Eng. 4(2):155-164.

Fisher AW, RWS Foulser, and SG Goodyear. 1990. "Mathematical Modeling of Foam Flooding.” SPE Paper 20195, 1990 SPE/DOE Seventh Symposium on Enhanced Oil Recovery, Tulsa, Oklahoma, April 22-25.

Fokas AS, and YC Yorsos. 1982. "On the Exactly Solvable Equation Occurring in Two-Phase Flow in Porous Media.” SIAM J. Appl. Math., 42:318-332.

Friedmann F, WH Chen, and PA Gauglitz. 1991. "Experimental and Simulation Study of HighTemperature Foam Displacement in Porous Media.” Soc. Petr. Eng. Res. Eng. 6(1):37-45.

Gauglitz PA, F Friedmann, SI Kam, and WR Rossen. 2002. "Foam Generation in Homogeneous Porous Media.” Chem. Eng. Sci. 57, 4037-4052.

Gillis JV and CJ Radke. 1990. “A Dual Gas Tracer Technique Determining Trapped Gas Saturation During Steady Foam Flow in Porous Media,” Presented at the 65th SPE Annual Technical Conference, New Orleans, Louisiana, September 1990; paper SPE 20519.

Hanson AT, B Dwyer, ZA Samani, and D York. 1993. "Remediation of Chromium-Containing Soils by Heap Leaching: Column Study.” Journal of Environmental Engineering. 119:825-841.

Hirasaki GJ and JB Lawson. 1985. "Mechanisms of Foam Flow in Porous Media: Apparent Viscosity in Smooth Capillaries.” Soc. Petr. Eng. J. 25(2):176-190.

Hirasaki GJ. 1989a. “A Review of the Steam Foam Process Mechanisms.” Paper SPE 19518, SPE, Richardson, Texas.

Hirasaki GJ. 1989b. “The Steam-Foam Process.” J. Petr. Technol. (May):44-456.

Hirasaki GJ, CA Miller, R Szafranski, JB Lawson, and N Akiya. 1997a. "Surfactant/Foam Process for Aquifer Remediation,” SPE 37257, in Proceedings of the SPE International Symposium on Oilfield Chemistry, Houston, Texas, Feb. 1997).

Hirasaki GJ, CA Miller, R Szafranski, JB Lawson, D Tanzil, RE Jackson, J Londergan, and H Meinardus. 1997b. "Field Demonstration of the Surfactant/Foam Process for Aquifer Remediation," SPE 39393, in Proceedings of the SPE Ann. Tech. Conf. and Exhibition, San Antonio, Texas, Oct. 1997). 
Huang CW and CH Chang. 2000. “A Laboratory Study on Foamenhanced Surfactant Solution Flooding in Removing Npentadecane from Contaminated Columns.” Coll. Surf. A: Physicochem. Eng. Aspects, 173, 171-179.

Hunt A. 2001. "Applications of Percolation Theory to Porous Media with Distributed Local Conductances.” Advances in Water Resources. 24(3-4):279-307.

Islam MR and SMF Ali. 1990. "Numerical Simulation of Foam Flow in Porous Media.” J. Can. Pet. Tech., 29(4):47-51.

Jeong SW, MY Corapcioglu, and SE Roosevelt. 2000. "Micromodel Study of Surfactant Foam Remediation of Residual Trichloroethylene.” Environ. Sci. Technol. 34, 3456-3461.

Jeong SW and MY Corapcioglu. 2003. "Physical Model Analysis of Foam-TCE Displacement in Porous Media.” AIChE Journal, 49(3):782-788.

Kam SI. 2008. "Improved Mechanistic Foam Simulation with Foam Catastrophe Theory. Colloids and Surfaces A: Physicochem.” Eng. Aspects, 318:62-77.

Kam SI, and WR Rossen. 2003. “A Model for Foam Generation in Homogeneous Porous Media.” SPE J., 8(4):417-425.

Kam SI, WW Frenier, SN Davies, and WR Rossen. 2007. "Experimental Study of High-Temperature Foam for Acid Diversion.” J. Pet. Sci. Eng. 58:138-160.

Kam SI, QP Nguyen, and A Li. 2007. "Dynamic Simulation with an Improved Model for Foam Generation.” SPEJ, 12(1):35-48.

Khatib ZI, GJ Hiraski, and AH Falls. 1988. "Effects of Capillary Pressure on Coalescence and Phase Mobilities in Forms Flowing Through Porous Media.” SPE Reser. Engr. (Aug.):919-926.

Khristov KHR, DR Exerowa, and P Krugljakov. 1981. Influence of Film Type on Foam Stability. Colloid J. USSR. 43:80-84.

Kibodeaux KR. 1997. Experimental and Theoretical Studies of Foam Mechanisms in Enhanced Oil Recovery and Matrix Acidization Applications. Ph.D. Dissertation, the University of Texas at Austin.

Kilbane II JJ, P Chowdiah, KJ Kayser, B Misra, KA Jackowski, VJ Srivastava, GN Sethu, AD Nikolov, DT Wasan, and TD Hayes. 1997. "Remediation of Contaminated Soils Using Foams.” Land Contam. Reclam. 5, 41-54.

Kommalapati RR, KT Valsaraj, WD Constant, and D Roy. 1998. "Soil Flushing Using Colloid Gas Aphron Suspensions Generated From a Plant-Based Surfactant.” J. Hazard. Mater. 60,73-87.

Kovscek AR. 1993. Foam Displacement in Porous Media: Experiment and Mechanistic Prediction by the Population Balance Method. PhD Thesis. University of California at Berkeley, Berkeley, California.

Kovscek AR. 1998. "Reservoir Simulation of Foam Displacement Processes.” The 7th UNITAR International Conference on Heavy Crude and Tar Sands, October 27-31, 1998, in Beijing, China. 
Kovscek AR, TW Patzek, and CJ Radke. 1995. “A Mechanistic Population Balance Model for Transient and Steady-State Foam Flow in Boise Sandstone.” Chemical Engineering Science, 50(23):3783-3799.

Kovscek AR, TW Patzek, and CJ Radke. 1997. "Mechanistic Form Flow Simulation in Heterogeneous and Multidimensional Porous Media.” Soc. Pet. Eng. J. 2:511-517.

Kovscek AR and CJ Radke. 1994. "Fundamentals of From Transport in Porous Media.” In: LL Schramm (Ed.), Foam: Fundamentals and Applications in the Petroleum Industry. American Chemical Society, Washington, D.C.

Kular G, K Lowe, and DA Coombe. 1989. "Foam Application in an Oil Sands Steam Flood Process,” SPE 19690, in Proceedings of the 64th Annual Technical Conf. of SPE, San Antonio, Texas, Oct 1989).

Larson RG and HT Davis. 1982. “Conducting Backbone in Percolating Bethe lattices.” J. Phys. C, $15: 2327$.

Law DHS, ZM Yang, and TW Stone. 1992. "Effect of the Presence of Oil on Foam Performance: A Field Simulation Study.” SPE Reservoir Eng. 7:228-236.

Liu D and WE Brigham. 1992. Transient Foam Flow in Porous Media with CAT Scanner.

DOE/BC/14600-19. Stanford University, Petroleum Research Institute, Stanford, California.

Longe TA. 1989. Colloidal Gas Aphrons: Generation, Flow Characterization, and Application in Soil and Groundwater Decontamination. Ph.D. Dissertation. Virginia Polytechnic Institute and State University, Blacksbury, Virginia.

Luthy RG, DA Dzombak, CA Peters, SB, Roy, A Romaswami, DV Nakles, and BR Nott. 1994. "Remediating Tar-Contaminated Soils at Manufactured Gas Plant Sites.” Environ. Sci. Technol. 28:266-276.

Marfoe CH, H Kazemi, and WF Ramirez. 1987. "Numerical Simulation of Foam Flow in Porous Media," SPE 16709, in Proceedings of the 62nd SPE Annual Meeting, Dallas, Texas, September 1987).

Marsden, SS. 1986. Foams in Porous Media. U.S. Department of Energy Report No. DE-AC0381SF11564. Stanford U. Petroleum Research Inst., Stanford, California.

McWhorter DB and DK Sunada. 1990. “Exact Integral Solutions for Two-Phase Flow.” Water Resource Res. 26:399-413.

McWhorter DB and BH Kueper. 1996. "Mechanics and Mathematics of the Movement of Dense NonAqueous Phase Liquid (DNAPLs) in Porous Media.” In Pankow JF and JA Cherry (eds.) Dense Chlorinated Solvents and other DNAPLs in Groundwater. Waterloo Press, Portland, Oregon.

Mohammadi SS and DA Coombe. 1992. "Characteristics of Steam/Foam Drive Process in Massive MultiZone and Thin Single Zone Reservoirs,” SPE 24030, in Proceedings of the SPE California Regional Meeting, Bakersfield, California, April 1992).

Mohammadi SS, DA Coombe, and VM Stevenson. 1993. “Test of Steam-Foam Process for Mobility Control in S. Casper Creek Reservoir,” J. Can. Pet. Tech., 32(10):49-54. 
Mulligan CN and BF Gibbs. 1993. Factors influencing the economics of biosurfactants. In: Marcel Dekker (Ed.), Surfactant Science Series, Biosurfactants: Production, Properties and Applications, Vol. 48. Marcel Dekker, Inc., New York, pp. 329-371.

Mulligan CN, RN Yong, and BF Gibbs. 2001a. "Surfactant Enhanced Remediation of contaminated Soil: a Review.” Eng. Geol. 60, 371-380.

Mulligan CN and F Eftekhri. 2003. "Remediation with Surfactant Foam of PCP-Contaminated Soil.” Engineering Geology 70: 269-279.

Mulligan CN and S Wang. 2006. "Remediation of a heavy metal-contaminated soil by a rhamnolipid foam.” Engineering Geology 85:75-81.

Mukerjee P and KJ Mysels. 1971. Critical micelle concentrations of aqueous surfactant systems. NSRDS-NBS 36, National Bureau of Standards, Washington.

Myers D. 1999. Surfaces, Interfaces, and Colloids: Principles and Applications, third ed. Wiley-Vch, New York.

Myers TJ and CJ Radke. 2000. "Transient Foam Displacement in the Presence of Residual Oil: Experiment and Simulation Using a Population-Balance Model.” Ind. Eng. Chem. Res. 39:2725-2741.

Patzek TW. 1988. "Description of Foam Flow in Porous Media by the Population Balance Approach,” Surfactant-Based Mobility Control: Progress in Miscible-Flood Enhanced Oil Recovery, DH Smith, Editor, American Chemical Society, Washington, D.C., p. 326-341.

Peters RW, CD Montemagno, and L Shem. 1992. "Surfactant Screening of Diesel-Contaminated Soil." Haz. Waste and Haz. Materials, 9:113-136.

Peters RW, WV Enzien, JX Bouillard, JR Frank, VJ Srivastava, JJ Kilbane II, and TD Hayes. 1994. "Nonaqueous-Phaseliquids-Contaminated Soil/Groundwater Remediation Using Foams in the In-Situ Remediation.” In: GW Gee and NR Wing (Eds.), Scientific Basis for Current and Future Technologies. Battelle Press, Columbus, Ohio, pp. 1067-1087.

Pope GA. 1980. “The Application of Fractional Flow Theory to Enhanced Oil Recovery”. Soc. Pet. Eng. J.,10,191-205.

Rajagopalan R and C Tien. 1976. "Trajectory Analysis of Deep-Bed Filtration with the Sphere-In-Cell Porous Media Model.” AIChE J. 22:523-533.

Ransohoff TC, PA Gauglitz, and CJ Radke. 1987. "Snap-off of Gas Bubbles in Smoothly Constricted Noncircular Capillaries.” AICHE Journal, 33(5):753-765.

Rosen MJ. 1989. Surfactants and interfacial phenomena, $2^{\text {nd }}$ ed. Wiley, New York.

Rosman A and SI Kam. 2009. "Modeling Foam-diversion Process Using Three-phase Fractional Flow Analysis in a Layered System.” Energy Sources, Part A: Recovery, Utilization, and Environmental Effects. 31(11):936-955.

Rossen WR. 1988. “A New Percolation Statistic with Unusual Properties.” J. Phys. A, 21:L533. 
Rossen WR. 1990a. "Theory of Mobilization Pressure Gradient of Flowing Foams in Porous Media. I. Incompressible Foam.” J. Colloid Interface Sci. 136(1):1-16.

Rossen WR. 1990b. "Theory of Mobilization Pressure Gradient of Flowing Foams in Porous Media. II. Effects of compressibility.” J. Colloid Interface Sci. 136(1):17-37.

Rossen WR. 1990c. "Theory of Mobilization Pressure Gradient of Flowing Foams in Porous Media. III. Asymmetric Lamella Shapes.” J. Colloid Interface Sci. 136(1):38-53.

Rossen WR. 1990d. "Minimum Pressure Gradient for Foam Flow in Porous Media: Effect of Interactions with Stationary Lamellae.” J. Colloid Interface Sci. 139(2):457-468.

Rossen WR and J Bruining. 2007. “Foam Displacements with Multiple Steady States.” SPE J, 5-18.

Rossen WR and PA Gauglitz. 1990. "Percolation Theory and Mobilization of Foams in Porous Media." Am. Inst. Chem. Eng. J. 36(8):1176-1188.

Rossen WR, JX Shi, and SC Zeilinger. 1994. "Percolation Modeling of Foam Generation in PorousMedia.” AICHE J. 40:(6):1082-1084.

Rossen WR and MW Wang. 1999. “Modeling Foams for Acid Diversion.” SPE J. 92-100 (June).

Rossen WR and ZH Zhou. 1995. "Modeling Foam Mobility at the Limiting Capillary Pressure.” SPE Adv. Technol. 3(1):146-153.

Rossen WR, SC Zeilenger, JX Shi, and MT Lim. 1994. "Mechanistic Simulation of Foam Processes in Porous Media," SPE 28940, in Proceedings of the 69th Ann. Tech. Conf. and Exhibition of the SPE, New Orleans, Louisiana, Sept 1994).

Rossen WR, SC Zeilenger, JX Shi, and MT Lim. 1999. "Simplified mechanistic simulation of foam processes in porous media.” SPE J. 4, 279-287 (September).

Rothmel RK, RW Peters, E St. Martin, and MF Deflaun. 1998. "Surfactant Foam/Biodegradation of In Situ Treatment of TCEDNAPLs.” Environ. Sci. Technol. 32:1667-1675.

Roy D, K-T Valsaraj, WD Constant, and M Darji. 1994. "Removal of Hazardous Oily Waste from a Soil Matrix Using Surfactants and Colloidal Gas Aphron Suspensions Under Different Flow Conditions.” Journal of Hazardous Materials 38:127-144.

Roy D, RR Kommalapati, KT Valsaraj, and WD Constant. 1995a. "Soil Flushing of Residual Transmission Fluid: Application of Colloidal Gas Aphron Suspensions and Conventional Surfactant Solutions.” Wat. Res. 29(2):589-595.

Roy D, S Kongaraa, KT Valsaraj. 1995b. "Application of surfactant solutions and colloidal gas aphron suspensions in flushing naphthalene from a contaminated soil matrix.” Journal of Hazardous Materials 42:247-263.

Schramm LL (Ed.). 1994. Foam: Fundamentals and Applications in the Petroleum Industry. America Chemical Society, Washington, D.C. 
Schramm LL. 2005. Emulsions, Foams, and Suspensions - Fundamentals and Applications. Wiley-VCH Verlag GmbH \& Co. KGaA, Weinheim, Germany.

Schramm LL and F Wassmuth. 1994. In LL Schramm (Ed.), Foam: Fundamentals and Applications in the Petroleum Industry. America Chemical Society, Washington, D.C.

Shi JX and WR Rossen. 1996. "Simulation and Dimensional Analysis of Foam Processes in Porous Media," SPE 35166, in Proceedings of the Permian Basin Oil and Gas Conf., Midland, Texas, Mar. 1996).

Smith DH. (ed.). 1988. Surfactant-Based Mobility Control: Progress in Miscible-Flood Enhanced Oil Recovery. ACS Symp. Ser. No. 373, Am. Chem. Soc., Washington, D.C.

Tang GQ and AR Kovscek. 2006. “Trapped Gas Fraction during Steady-State Foam Flow.” Transport in Porous Media, 65:287-307.

Tanzil D, GJ Hirasaki, and CA Miller. 2002. "Mobility of Foam in Heterogeneous Porous Media: Flow Parallel and Perpendicular to Stratification.” Soc. Petrol. Eng. J. 7:203-212.

Vignon BW and AJ Rubin. 1989. Practical Considerations in the Surfactant-Aided Mobilization of Contaminants in Aquifers. J. Water Pollut. Control. Fed., 61:1233-1240.

Wan J, S Veerapaneni, F Gadelle, and TK Tokunag. 2001. "Generation of Stable Microbubbles and Their Transport Through Porous Media.” Water Resources Research, 37(5):1173-1182.

Wang S, and CN Mulligan. 2004. "Rhamnolipid Foam Enhanced Remediation of Cadmium and Nickel Contaminated Soil.” Water Air Soil Pollut. 157, 315-330.

Welge HJ. 1952. “A Simplified Method for Computing Oil Recovery by Gas or Water Drive.” Trans. AIME 195:91-98.

Yao KM, MT Habibian, and CR O’Melia. 1971. “Water and wastewater filtration: Concepts and Applications.” Environ. Sci. Technol. 5:1105-1112.

Zhou ZH and WR Rossen. 1995. "Applying Fractional-Flow Theory to Foam Processes at the Limiting Capillary Pressure.” SPE Adv. Technol. 3(1):154-162. 

Appendix A

Glossary 



\section{Appendix}

\section{Glossary}

An (S) following the definition indicates from Schramm and Wassmuth (1994); a (KR) indicates from Kovscek and Radke (1994).

Black film - Fluid films yield interference colors in reflected white light that are characteristic of their thickness. At a thickness of about $0.1 \mu \mathrm{m}$, the films appear white and are termed silver films. At reduced thicknesses, they first become grey and then black (black film). Among thin equilibrium (black) films, one may distinguish those that correspond to a primary minimum in interaction energy, typically at about 5-nm thickness (Newton black films), from those that correspond to a secondary minimum, typically at about 30-nm thickness (common black films). (S)

Bubble division - A mechanism for bubble generation in porous media. Lamellae or bubbles are divided into more lamellae or bubbles. Mobile foam must pre-exist. (KR)

Bulk foam -foam is designated as bulk when the pore size is much larger than the size of individual foam bubbles. (KR)

Confined foam -foam is designated as confined foam when the pore size of the medium is comparable or less than the size of the dispersed gas bubbles and the bubbles and lamellae span completely across pores. (KR)

Continuous-gas foam - the medium contains one or several interconnected gas channels that are uninterrupted by lamellae over macroscopic distances. (KR)

Cosurfactant - Any chemical, whether surface active by itself or not, that may be added to a system to enhance the effectiveness of a surfactant. (S)

Critical film thickness - A fluid film may thin to a narrow range of film thicknesses within which it either becomes metastable to thickness changes (equilibrium film) or else ruptures. Persistent foams comprise fluid films at their critical film thickness. (S)

Critical micelle concentration (CMC) - The surfactant concentration above which molecular aggregates, termed micelles, begin to form. In practice, a narrow range of surfactant concentrations represents the transition from a solution in which only single, unassociated surfactant molecules (monomers) are present to a solution containing micelles. (S)

Discontinuous-gas foam - the entire gas phase is made discontinuous by lamellae, and there are no gas channels that are continuous. (KR) 
Disjoining pressure - The negative derivative, with respect to distance, of the Gibbs energy of interaction per unit area yields a force per unit area between colloidal species, termed the disjoining pressure. Example: In a thin liquid film, the disjoining pressure equals the pressure, beyond the external pressure, that must be applied to the liquid in the film in order to maintain a given film thickness. (S)

Effective foam viscosity - For foam flowing in porous media, the foam's effective viscosity is that calculated from Darcy's law. This value is an approximation because foams are compressible and are also usually non-Newtonian. (S)

Film - Any layer of material that covers a surface and is thin enough to not be significantly influenced by gravitational forces. (S)

Film drainage - The drainage of liquid from a lamella of liquid separating droplets or bubbles of another phase (i.e., in a foam or emulsion). Also termed thin film drainage. (S)

Foam - A gas phase dispersed within a liquid phase and stabilized by surfactant adsorbed at the gas/liquid interface. Foam can be aqueous or non-aqueous. (KR)

Foam drainage - See film drainage.

Foam quality - the volume fraction of gas in foam. (KR)

Foam texture - the number of lamellae per unit volume of foam; is used to quantify the bubble population. (KR)

Foaming agent - Any agent that stabilizes a foam. The foaming agent may make it easier to form a foam and provide stability against coalescence. Foaming agents are usually surfactants. Also termed foam booster, whipping agent, and aerating agent. (S)

Foaming power - In foaming, 100 times the ratio of gas volume to liquid volume in a foam. (S)

Gas emulsion - "Wet" foams in which the liquid lamellae have thicknesses on the same scale or larger than the bubble sizes. Typically in these cases, the gas bubbles have a spherical rather than polyhedral shape. Other synonyms include gas dispersion and Kugelshaum. (S)

Kraft point - The temperature above which the solubility of a surfactant increases sharply (micelles begin to form). (S)

Kugelschaum (“ball foam”) - Foam that consists of well-separated, spherical bubbles. (KR)

Leave-behind - A mechanism for bubble generation in porous media. As two gas menisci invade adjacent liquid-filled pore bodies, a lens of liquid is left behind as the two menisci converge downstream. Lenses created by Leave-behind do not make the gas phase discontinuous. (KR) 
Leverett J-function - A dimensionless function of water saturation describing the capillary pressure (Leverett 1941). It is defined as $J\left(S_{w}\right)=\frac{p_{c}\left(S_{w}\right) \sqrt{k / \phi}}{\sigma \cos \theta}$, where $S_{w}$ is the water saturation measured as a fraction, $\mathrm{p}_{\mathrm{c}}$ is the capillary pressure, $\mathrm{k}$ is the permeability, $\phi$ is the porosity, $\sigma$ is the surface tension, and $\theta$ is the contact angle. The function is constant for a given saturation within a reservoir, thus relating reservoir properties for neighboring beds. The Leverett J-function is an attempt at extrapolating capillary pressure data for a given rock to rocks that are similar but with differing permeability, porosity, and wetting properties. It assumes that the porous rock can be modeled as a bundle of non-connecting capillary tubes, where the factor is a characteristic length of the capillaries' radii. For further discussion on the Leverett J-function, see http://www.ux.uis.no/ s-skj/ResTek1v03/Notater/Tamu.Lecture.Notes/Capillary.Pressure/Lecture 16.ppt.

Limiting capillary pressure - The capillary above which lamellae cannot be sustained. The corresponding saturation is called limiting water saturation. (KR)

Micelle - An aggregate of surfactant molecules in solution. (S)

Mobility reduction factor - A dimensionless measure of the effectiveness of a foam at reducing gas mobility when flowing in porous media. The mobility reduction factor is equal to the mobility (or pressure drop) measured for foam flowing through porous media, divided by the mobility (or pressure drop) measured for surfactant-free solution and gas flow at the same volumetric flow rates. (S)

Newtonian fluid - A fluid whose rheological behavior is described by Newton's law of viscosity (shear stress is proportional to the shear rate). The proportionality constant is the coefficient of viscosity. (S)

Non-Newtonian fluid - A fluid whose viscosity varies with applied shear rate (flow rate). Non-Newtonian flow refers to fluid flow that does not obey Newton's law of viscosity. (S)

Polyderschaum (“polyhydral foam”) - Foam that consists of polyhedral bubbles separated by surfactantstabilized thin-liquid films called lamellae. (KR)

Shear-thinning fluid - a fluid in which viscosity decreases with increasing rate of shear.

Snap-off - A mechanism for bubble generation in porous media. A bubble is formed when the gas pushes the gas-liquid interface through a pore throat and then the interface is snapped off. Snap-off creates gas bubbles that are approximately the size of pore bodies. Discontinuous-gas foam is created by snap-off. It can result in a several-hundred fold reduction in gas mobility. (KR)

Strong foam - Finely textured foam (large number of lamellae with a small bubble size). It reduces gas mobility remarkably (KR)

WAG - Water-Alternating-Gas displacement

Weak foam - Coarse textured foam (small number of lamellae with a large bubble size). It provides a moderate reduction in gas mobility (KR) 


\section{References}

Kovscek AR and CJ Radke. 1994. "Fundamentals of From Transport in Porous Media.” In: LL Schramm (Ed.), Foam: Fundamentals and Applications in the Petroleum Industry. American Chemical Society, Washington, D.C.

Leverett MC. 1941. “Capillary Behaviour in Porous Solids.” Transactions of the AIME, 142:159-172.

Schramm LL and F Wassmuth. 1994. In LL Schramm (Ed.), Foam: Fundamentals and Applications in the Petroleum Industry. America Chemical Society, Washington, D.C. 


\section{Distribution}

No. of

Copies

\# Name

DOE EM-32

Skip Chamberlain PDF

Ming Zhu PDF

Amec Geomatrix Consultants, Inc.

Dawn Kaback

PDF

MSE Technology Applications, Inc.

Jody Bickford

Marty Foote

PDF

PDF

Savannah River National Laboratory

Dan Kaplan

PDF

Idaho National Laboratory

Mark Ankeny

PDF

Larry Hull

PDF

Roelof Versteg

PDF

Oak Ridge National Laboratory

Baohau Gu

PDF

Lawrence Berkeley National Laboratory

Susan Hubbard

PDF

11 Pacific Northwest National Laboratory

4 DM Wellman

BWO

MD Freshley

K9-33

ZF Zhang

K9-33

WJ Martin

K8-46

SV Mattigod

K3-62

TJ Gilmore

K6-96

L Zhong

K6-96

VL Freedman
No. of

Copies 


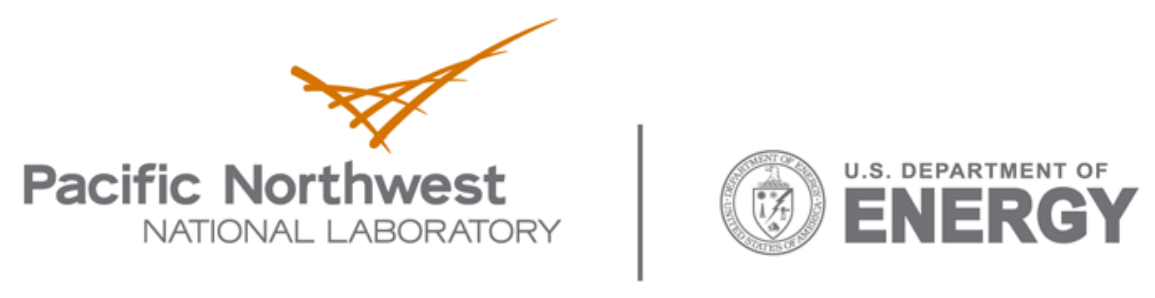

902 Battelle Boulevard

P.O. Box 999

Richland, WA 99352

1-888-375-PNNL (7665)

www.pnl.gov 